\title{
Wolfgang Däubler Bundesdeutsches Arbeitsrecht in der ehemaligen DDR
}

\section{Einleitung}

Art. 8 des Vertrages "über die Herstellung der Einheit Deutschlands « ${ }^{1}$ trifft eine eindeutige Entscheidung: Mit dem Wirksamwerden des Beitritts, d. h. ab 3. 10. 1990 tritt im Gebiet der ehemaligen DDR das gesamte Bundesrecht in Kraft. Anlage I sieht für eine Reihe von Vorschriften Modifikationen vor; die (sehr viel kleinere) Anlage II nennt jene Bestimmungen des DDR-Rechts, die für einige Zeit oder unbefristet weitergelten. Eine Anlage III, die einzelne Teile des DDR-Rechts mit oder ohne Veränderungen auf die Bundesrepublik erstreckt hätte, existiert nicht. Eine Anlage IV, die insoweit einzelnen Normen des Bundesrechts die Weiterexistenz garantiert hätte, war erst recht entbehrlich. Die Herstellung der Rechtseinheit war ein asymmetrischer Vorgang.

Die Beseitigung des DDR-Rechts kennt kaum Parallelen. So galt Recht aus der Zeit des Faschismus der Jahre 1933 bis 1945 weiter, sofern es mit dem Grundgesetz. vereinbar war. ${ }^{2}$ Auch die DDR hatte insoweit keinen totalen Trennungsstrich gezogen, allerdings verlangt, daß die Normen des »bürgerlichen Gesetzgebers« durch ein Staatsorgan »sanktioniert», d. h. für verfassungskonform befunden wurden; dies konnte nicht nur durch den Gesetzgeber, sondern z. B. auch durch eine gerichtliche Entscheidung erfolgen. ${ }^{3}$ Auch im Verhältnis zwischen Deutschland und Frankreich war man bei Grenzverschiebungen weniger radikal. In den linksrheinischen Gebieten galt der französische code civil bis $1900^{4}$, in Elsaß-Lothringen gelten noch heute zahlreiche Normen aus der Zeit vor 19 r $^{3}$, nicht zuletzt auch im Arbeitsrecht ${ }^{6}$. Wenn man eine äußerliche Parallele sucht, findet man sie am ehesten in der pauschalen Ablösung des zaristischen Rechts nach der russischen Oktoberrevolution. ${ }^{7}$ Inhalt-

I Vom 31.8. 1990, BGBI. II, 889, im folgenden: Einigungsverrag.

2 Are. 123 GG.

3 Einzelheiten und Nachweise bei Pfarr, Auslegungstheorie und Auslegungspraxis im Zivil- und Arbeitsrechi der DDR, Berlin 1972, S. $35 \mathrm{ff}$

4 Vgl. Grimm RabelsZ so (1986), 61, 69.

s Überblick bei Woehrling BWVBL. 1983,386 sowie bei: Institut du droit local (éd.), Histoire du droit local, Actes du Colloque du 19 octobre 1989, Strasbourg 1990 (u. a. Grundbuchrecht, ProzeBrecht, Jagdrecht, Kommunale Selbstverwaltung).

6 Simon, Le droit local du travail applicable en Alsace-Moselle: Repos dominical, Garantie de Ressources, Clause de non-concurrence, Durée du préavis, Strasbourg 1990. S. weiter das zweisprachige Buch: Institut du droit local (Hrsg.), Drott du travail/Arbeitsreche, Referate zum Kolloquium vorn $1_{4}$. Märe rg91, Strasbourg 1991. Dazu Kraushaar AuR 1991, i45.

7 Dekret über die Volksgerichte vom 30.11.1918, in dem bestimmt war: „Bei der Entscheidung von Gerichessachen wendet das Volksgericht die Dekrete der Arbeiter- und Bauernregierung an, und im Fall des Fehlens eines entsprechenden Dekrets oder seiner Unvoliständigkeit laßt es sich von dem sozıalistischen Rechisbewußtsein leiten.

Anmerkung: Der Verweis in Entscheidungen oder Urteilen auf die Gesetze gesturzter Regierungen ist verboten, zitiert nach Reich, Sozialismus und Zivilrecht, Frankfurt/Main 1972, S. $9411 ., 103$. 
lich ist der Vorgang völlig neuartig: Soweit ersichtlich, hat noch nie ein industrialisiertes Land pauschal die Rechtsordnung eines anderen übernommen. Die "parallele Welt« ist zusammengebrochen.

Im Folgenden soll dieser Prozeß am Beispiel des Arbeitsrechts näher beschrieben werden. Wie sehen die verbliebenen Reste aus? Was geschah mit Kollektiv- und mit Arbeitsverträgen aus der Zeit vor der deutschen Einheit? (Dazu unten II). Weiter ging es ja um mehr als einen Austausch von Rechtsordnungen. Der Systemwandel, die Ersetzung der "realsozialistischen" durch die bürgerlich-demokratische Ordnung erforderte eine Art Sonderrecht, um verbliebene Herrschaftspositionen des "Ancien Régime « abzubauen. Dieses "Entsozialisierungsrecht « betraf das Eigentum, insbes. aber auch den öffentlichen Dienst und die Behandlung der Renten solcher Personen, denen eine besondere "Staatsnähe unterstellt wurde (unten III).

Der »Export « des bundesdeutschen Rechts sah und sieht sich mit einer Reihe von Problemen konfrontiert. Würden nicht vernünftige Regelungen des alten Rechts die Unterstützung der Bevölkerung behalten, würde nicht die "Überstülpung « eines neuen zu Protest führen? Würde es möglich sein, in gleicher Weise wie in der alten Bundesrepublik Gewerkschaften, Arbeitgeberverbände und Betriebsrätc zu schaffen? Was bedeutet es für den einzelnen Bürger wie für Rechtsanwälte und Gerichte, von einem Tag auf den anderen mit einem völlig neuartigen Recht arbeiten zu nüssen? (Unten IV).

eben dieser rechtssoziologischen Perspektive interessiert das Funktionieren industrieller Beziehungen unter Krisenbedingungen. Im verarbeitenden Gewerbe sank die Nettoproduktion zwischen drittem Quartal 1990 und drittem Quartal I991 um 40 Prozent, ${ }^{8}$ seit der "Wende " ist ein Rückgang um rund zwei Drittel zu verzeichnen. ${ }^{9}$ Über 30 Prozent der Erwerbstätigen sind ohne Beschäftigung. ${ }^{10}$ Wie vollzieht sich Tarifpolitik unter solchen Bedingungen, die in der alten Bundesrepublik niemals aufgetreten waren? Entsteht auch in der Bundesrepublik eine Art Notstandsarbeitsrecht, ein «diritto del lavoro di emergenza«, welche Formen der Staatsintervention werden entwickelt? (Unten V).

\section{Die Ausdehnung des bundesdeutschen Arbeitsrechts}

\section{Die erste Etappe: Der Staatsvertrag vom 18. Mai $1990^{\prime \prime}$}

Durch den Staatsvertrag »über die Schaffung einer Währungs-, Wirtschafts- und Sozialunion zwischen der Bundesrepublik Deutschland und der DDR « wurde mit Wirkung vom 1.7. 1990 das gesamte kollektive Arbeitsrecht der Bundesrepublik in die damals noch bestehende DDR übernommen. Koalitionsfreiheit, Tarifautonomie, Arbeitskampfrecht, Betriebsverfassungsrecht und Unternehmensmitbestimmung galten von da an in gleicher Weise wie in der Bundesrepublik. Dazu kamen das Kündigungsschutzgesetz sowie die für Arbeiter geltenden Kündigungsfristen, die in der DDR auf alle Arbeitnehmer erstreckt wurden. Zugrunde lag die berechtigte Überlegung, daß die frühere arbeitsrechtliche Ordnung mit ihrer zentralistischen Grundstruktur und der Garantie des Rechts auf Arbeit' ${ }^{\prime 2}$ für die neuen marktwirt-

\footnotetext{
8 Bispinck/WSI-Tarifarchiv WSI-Mitt. 1992, 121.

9 Kromphardt/Bruno-Latocha GMH 1991, 734.

so Zahlenangaben unten $\mathrm{V}$ t

11 BGBI. II, $\{37$.

12 Dazu etwa Kunz WSI-Mirt. 1990, 308 ff.; ders., Des Menschen Recht auf Arbeit, Berlin 1989. Zu dem
} 
schaftlichen Bedingungen ungeeignet war. Das Arbeitsgesetzbuch der DDR wurde

einer grundlegenden Änderung unterzogen'3.

Gleichwohl blieben zahlreiche wichtige Besonderheiten: Die Kündigungsschutzvorschriften zugunsten Alleinerziehender, die Vorschriften zum Schutze der erwerbstätigen Frau, die Regeln über die Haftung im Arbeitsverhältnis sowie das Arbeitsschutzrecht galten weiter. Das gerichtliche Verfahren blieb unangetastet. Auch die außerhalb des Justizsystems stehenden Konfliktkommissionen bestanden fort, erfuhren jedoch eine Umgestaltung. Bei ihnen handelte es sich um von den Belegschaften auf Vorschlag der Gewerkschaft gewählte Ausschüsse, die bei allen arbeitsrechtlichen Konflikten automatisch angerufen werden mußten; nur wenn es dort nicht gelang, einen allseits akzeptierten Kompromiß zu finden, konnten die Gerichte eingeschaltet werden'4. Durch DDR-Gesetz vom 29. Juni 1990's wurden sie in "Schiedsstellen « umgewandelt, die "paritätisch « mit einem Arbeitgeber- und einem Belegschaftsvertreter sowie einem neutralen Vorsitzenden besetzt sind. An ihrer grundsätzlichen Funktion als "Filter « änderte sich zunächst nichts. ${ }^{16}$

\section{Einigungsvertrag vom $3 x$. August $1990^{17}$}

Diese verbliebenen Teile des DDR-Arbeitsrechts wurden durch den Einigungsvertrag fast völlig beseitigt. Die Tatsache, daß es sich nicht um »Erblasten «, sondern um durchaus marktwirtschaftskonforme Bestimmungen handelte, spielte keine Rolle. Als wichtige Punkte seien genannt:

- Nach $\mathbb{\$} 40$ ff. AGB-DDR bedurfte der Arbeitsvertrag wie die Kündigung der Schriftform. Dieses Erfordernis ist durch die Übernahme der $\$ 6$ I ff. BGB ersatzlos weggefallen.

- Nach $₫ 85$ AGB waren Versetzungen, die über 4 Wochen hinausgingen, nur mit Zustimmung des Arbeitnehmers zulässig. Eine arbeitsvertragliche "Ermächtigung " reichte nicht. Auch dies ist nunmehr gegenstandslos.

- Nach $₫ 5^{8}$ Abs. Ib AGB bestand ein Sonderkündigungsschutz für alleinerziehende Arbeitnehmer mit Kindern bis zu 3 Jahren. Eine ordentliche Kündigung ist bei diesem Personenkreis nur im Falle der Betriebsstillegung zulässig; außerdem muß auch dann - ebenso wie bei der fristlosen Kündigung - das Arbeitsamt zustimmen. Die Regelung gilt nur noch für solche Arbeitnehmer weiter, deren Kind vor dem 1. Januar 1992 geboren wurde.

- Nach der Geburt eines Kindes hatten Frauen gem. $\$ 244$ Abs. I AGB Anspruch auf Wochenurlaub für die Dauer von 20 Wochen. Nunmehr gilt die achtwöchige Schutzfrist nach $\$ 6$ Abs. I Mutterschutzgesetz.

- Nach $\$ 246$ Abs. x AGB waren Mütter auf Verlangen nach dem Wochenurlaub bis zum Ende des ersten Lebensjahres des Kindes von der Arbeit freizustellen. War kein Krippenplatz verfügbar, konnte die Freistellung längstens bis zum Ende des 3. Lebensjahres des Kindes verlängert werden ( $\$ 246$ Abs. 2 AGB). Während dieser

Übergangsrecht, das die Volkskammer noch vor den freien Wahlen am 18.3.1990 beschlossen hatte und das alsbald wiederaufgehoben wurde, s. Däubler AuR 1990, $149 \mathrm{fl}$. Bemerkenswert war insbes. das Gewerkschaftsgesetz vom 6.3.1990 (GBI. 1, 110 ), eine Art *Gesetz zur Herstellung des Gewerkschaftsparadieses $\star$, das den realen Verhailnissen in keiner Weise Rechnung trug (vgl. Bobke WSI-Mitt. 1990, $312 \mathrm{ff}$.).

13 Änderungsgesetz vom 22.6. 1990, GBI. I, 371 .

${ }_{14}$ Berg NZA 1990, 19; Otte-Sahr-Herzog (Hrsg.), Die Konfliktkommission - Ein Leiffaden, 3. Aufl., Berlin 1988.

is GBI. 1, sos.

16 Einzelheiten bei Däubler, Ratgeber Arbeitsrucht. Mit den Übergangstegelungen fur die neuen Bundesländer, Reinbek 1991, S. 499 ff.

17 BGBI. II, 889. 
Zeit wurde eine Lohnersatzleistung gewährt. Nach dem nunmehr geltenden BErzGG war der Erziehungsurlaub zunächst auf $x 8$ Monate beschränkt; erst durch Gesetz vom 6. Dezember $1991^{18}$ wurde er auf drei Jahre verlängert, sofern das Kind nach dem 31. 12. 1991 geboren ist ( $\$$ is Abs. I BErzGG n. F.). Erziehungsgeld wird weiter für höchstens 18 Monate gezahlt; erst für Kinder, die nach dem 31. 12. 1992 geboren werden, beträgt die Frist zwei Jahre $(\$ 4$ Abs. I BErzGG n.F.).

- Nach $\$ 186$ Abs. I AGB waren Arbeitnehmer von der Arbeit freizustellen, »wenn es zur ärztlich bescheinigten Pflege ihres erkrankten Kindes oder zum Arztbesuch ihres Kindes erforderlich" war. Abs. 2 derselben Vorschrift sah eine Lohnersatzleistung durch die Sozialversicherung vor. Diese Regelung lief am 30. Juni 1991 aus. ${ }^{19}$ Nach dem Recht der Bundesrepublik bestand zunächst gemäß $\$ 6 \times 6$ Abs. I BGB Anspruch auf bezahlte Freistellung in Höhe von höchstens s Arbeitstagen pro Jahr, soweit das Kind nicht älter als 8 Jahre war. ${ }^{20}$ Der durch Gesetz vom 20. 12. $1991^{21}$ geänderte $\ 45$ SGB V sieht nunmehr unbezahlte Freistellung von ro Arbeitstagen pro Kind (höchstens 2s Arbeitstage im Kalenderjahr) und die Gewährung von Krankengeld vor; Voraussetzung ist, daß das Kind noch nicht $\mathrm{I} 2$ Jahre alt ist und keine andere Pflegeperson zur Verfügung steht. ${ }^{22}$ Vermutlich wird das BAG die neue Altersgrenze in seine Rechtsprechung zu $\$ 6 x 6$ Abs. x BGB übernehmen.

- Nach $₫ 774$ AGB durften an zwei aufeinanderfolgenden Tagen nicht mehr als insgesamt 4 Überstunden geleistet werden. Nur bei Notfällen bestand eine Ausnahme. Diese Vorschrift ist ersatzlos weggefallen.

- Aufrechterhalten blieb der Sonderkündigungsschutz der $»$ Kämpfer gegen den Faschismus und der Verfolgten des Faschismus« nach $\$ \$ 8 \mathrm{AGB}$, also für eine Gruppe, die 47 Jahre nach Ende des Zweiten Weltkriegs kaum mehr im Erwerbsleben stehen dürfte. Erstaunlicherweise hat man nicht versucht, den Opfern des Stalinismus einen vergleichbaren Schutz zu gewähren.

- Das gerichtliche Verfahren bestimmt sich nunmehr nach dem ArbGG. Aufrechterhalten blieb das Schiedsstellengesetz ${ }^{23}$, dessen praktische Wirksamkeit sehr unterschiedlich eingeschätzt wird. ${ }^{24}$ Große Erfahrungen konnte man allerdings nicht sammeln: Durch Gesetz vom 20. 12. x99 $\mathrm{x}^{25}$ wurde es zum 3 x. 12. 1992 außer Kraft gesetzt; anhängige Verfahren können zu Ende geführt werden. Die neuen Bundesländer sind ermächtigt, das Gesetz schon vorher auslaufen zu lassen, sofern sie gleichzeitig selbständige Arbeitsgerichte errichten.

- Das DDR-Arbeitsschutzrecht wurde ersatzlos beseitigt, obwohl die Unfallhäufigkeit erheblich unter der in der alten Buŕdesrepublik lag. ${ }^{26}$

- Dic Haftung des Arbeitnehmers war nach $\$ 261$ Abs. 2 AGB grundsätzlich der Höhe nach auf einen monatlichen Tariflohn beschränkt. Diese Regelung lief am

$18 \mathrm{BGBl} . \mathrm{I}, 21_{4} 2$.

19 Anlage II Kap. VIII Sachgebiet A Abschnitt III Nr. 1 e des Einigungsvertrags.

20 Nachweise zur Rechtsprechung, die diesen Anspruch aus $\$ 616$ Abs. 1 ableiter, bei Däubler, Das Arbeitsrecht 2, 7. Aufl., Reinbek 1990, S. 433.

2 I BGBI. I, 2325 .

22 Bei Alleinerziehenden verdoppeln sich die Sätze: 20 Tage pro Kind, insgesamt hóchscens so Tage. Vor der Novellierung sah $\$ 45 S G B$ V ganze's Tage pro Jahr vor und das nur dann, wenn das Kind noch keine 8 Jahre alt war.

23 S. oben Fn is.

24 Dazu Beck u. a., demnächst in: Festschrift Gnade, Köln 1992.

2) BGBI. I, 2321.

26 Angaben bei Motsch, Der Schutz der Werktatigen im Arbeitsrecht der DDR, in: Rottmann-RammWesten-Motsch, Die DDR im Lichte der Grundrechte und der Rechtsstaatsidee, Heidelberg 1989, S. 10 Iff. 
31.12.1991 aus ${ }^{27}$, das Recht der Bundesrepublik kennt eine entsprechende Rechtsnorm nicht.

Die offiziell gegebene Begründung ist wenig überzeugend. In einem einheitlichen Staat müsse eine einheitliche Arbeitsrechtsordnung gelten; weiter könnten sich günstigere Rechtspositionen für Arbeitnehmer in den neuen Bundesländern als Investitionshemmnis erweisen ${ }^{28}$. Dagegen spricht, daß das gesamte tarifliche Arbeitsrecht und damit ein großer Teil des effektiven Schutzstandards in Westdeutschland - nicht übernommen wurde ${ }^{29}$; bessere gesetzliche Regelungen hätten also nur anderweitige' Nachteile kompensiert. Auch hätten einige Sonderregelungen vermutlich weniger Kollisionsprobleme geschaffen als sie sowieso durch die Existenz zweier Wirtschaftszonen und eines unterschiedlichen Tarifniveaus gegeben sind.$^{30}$ Die totale Übernahme des bundesdeutschen Rechts läßt sich eher mit ideologischen Gründen erklären: Die Erinnerung an die Vergangenheit soll unwiderruflich ausgelöscht werden." Als weiteres Beispiel mag die Aufhebung des Gesetzes über internationale Wirtschaftsverträge vom 5 . Februar $1976^{32}$ stehen. Es regelte auf der Basis der Vertragsfreiheit $\left(\S_{4}\right)$ Verträge der Außenhandelsorganisationen der DDR mit ausländischen Unternehmen und kodifizierte zahlreiche Vertragstypen, die sich in der Praxis herausgebildet haben und die bislang nirgends im deutschen Recht geregelt sind. ${ }^{33}$ Weshalb dies von westlicher Seite nicht nur gefordert, sondern auch durchgesetzt wurde, soll uns an späterer Stelle interessieren. ${ }^{34}$ Die "Gegenleistung « des Art. 30 Einigungsvertrag, wonach das Arbeitsschutz- und das Arbeitsvertragsrecht sobald wie möglich zu kodifizieren sind ${ }^{35}$, ist wenig wert, da jedenfalls im Arbeitsvertragsrecht eine praktische Umsetzung in weiter Ferne liegt. ${ }^{36}$

\section{Aufrechterhaltung von Verträgen und Verwaltungsakten}

Während sich altes Gesetzesrecht im Prinzip durch neues ersetzen läßt, kann dies bei Verträgen und Verwaltungsakten nicht geschehen; andernfalls würde man ein totales Chaos provozieren. Der durch den Einigungsvertrag neu geschaffene Art. 232 EGBGB hält alle bestehenden Verträge einschließlich der Arbeitsverträge aufrecht; auf letztere findet lediglich ab 3. 10. I990 das Arbeitsrecht der Bundesrepublik Anwendung. Rahmenkollektivverträge, die zwischen den DDR-Gewerkschaften und den zuständigen Ministerien für die einzelnen Branchen verabredet wurden, bleiben solange in Kraft, bis sie durch Tarifverträge neuen Rechts abgelöst werden. ${ }^{37}$ Das-

27 Anlage II Kap. VIII Sachgebiet A Abschnitt III Nr. If des Einigungsvertrags.

28 Wlotzke-Lorenz Beilage 35/1990 zu BB S. 4 (beide Autoren - leitende Beamte im Bonner Arbeitsministerium - waren an der Ausarbeitung der entsprechenden Passagen des Einigungsvertrags beteilige).

29 Darauf verweist auch Kunz WSI-Mirc. 1990, 309. Vgl. weiter Plander in: Muszynski (Hrsg.), Deutsche Vereinigung, Gegenwartskunde - Sonderheft 7, $1991,158$.

30 Dazu unten V 2.

31 Auf derselben Linie liegr es, wenn z. B. den Sportern der ehemaligen DDR pauschal Doping-Praktiken vorgeworfen, wenn die rechtsstaatswidrigen Praktiken des Sraatssicherheitsdienstes in allen Details geschildert oder Korruptionsfälle ausgebreitet werden, ohne daß Vergleiche mit der alten Bundesrepublik oder anderen Ländern gezogen werden. Der Zufall will es, daß der nPalası der Republik $\alpha$, Sicz des DDR-Parlaments, wegen Asbestverseuchung gesperr ist und uber seinen Abriß diskutiert wird. 32 GBI. I, 6I.

$33 \mathrm{~S}$. ecwa den Anlagenvertrag nach $\$ 88 \mathrm{ff}$, den Kundendienstvertrag nach $\$ \oint_{129} \mathrm{ff}$, den Lizenzvercrag nach $\$ \$ 176 \mathrm{ff}$. und das Akkreditiv nach $\$ \$ 256 \mathrm{ff}$. des Gesetzes; vgl. auch die Besprechung des Kommentars von Maskow-Wagner durch Schanze (Rabels $Z_{45}, 1981,814$ ).

$34 \mathrm{~S}$. unten IV $\mathrm{t}$.

35 Dazu Mückenberger KJ 1990, $485 \mathrm{ff}$.

36 Zum Professorenentwurf eines Arbeitsvertragsgeseczes s. Däubler AuR 1992, I 29 ff. Grundsäczlich fur eine gesetzliche Regelung Kunz ZfA ig9 1, $376 f$.

37 Anlage I Kap. VIIl Sachgcbiet A Abschnitc III Nr. 14 zum Einigungsvertrag. 
selbe dürfte auch für betriebliche Regelungen gelten. ${ }^{38}$ Art. I9 des Einigungsvertrags ordnet die Fortgeltung von Verwaltungsakten wie z. B. einer Fahrerlaubnis an, was im Arbeitsrecht insbesondere Bedeutung für die Anerkennung als Schwerbehinderter hat. ${ }^{39}$

\section{Das Recht des Systemwechsels}

\section{x. Die Änderung der Eigentumsordnung}

\section{a) Privatisierung des Volkseigentums}

Nach DDR-Recht standen alle Industriebetriebe, aber auch weite Teile des sonstigen Produktions- und Dienstleistungsapparats in Volkseigentum. Dieses wurde de facto durch den Staat ausgeübt, der mit Hilfe der Planung die Produktion bis ins Detail hinein bestimmte. Die Wirtschaft war in einzelne Einheiten aufgegliedert (Kombinate - unseren Konzernen vergleichbar; VEB - Einzelunternehmen; sonstige Einrichtungen). Diese waren zwar selbständige juristische Personen, besaßen jedoch an den von ihnen genutzten Betriebsmitteln kein Eigentum, sondern nur ein Nutzungsund Verfügungsrecht im Rahmen der Planvorgaben. Dem Prinzip der Einzelleitung entsprechend ${ }^{\circ \circ}$ wurden die Kombinate von Generaldirektoren, die Betriebe von Betriebsdirektoren geleitet.

An dieser überkommenen Struktur setzten die ersten Reformversuche an. Am r. März 1990 erließ die noch maßgebend von der SED beeinflußte Modrow-Regierung die sog. Umwandlungsverordnungt' , die alle Kombinate, VEBs und vergleichbare Einheiten verpflichtete, sich in eine $A G$ oder $\mathrm{GmbH}$ umzuwandeln. Alleiniger Gesellschafter sollte die am selben Tage gegründete Treuhandanstalt werden; eine neugegründete $\mathrm{GmbH}$ hatte nicht mehr Rechte als der Vorgänger-VEB.4 Von den Möglichkeiten der Verordnung wurde nur sehr zögernd Gebrauch gemacht.

Die Situation änderte sich grundlegend, als das neue Parlament am 17.6. 1990 das "Gesetz zur Privatisierung und Reorganisation des volkseigenen Vermögens (sog. Treuhandgesetz) beschloß.43 Es stellt die bis heute entscheidende Rechtsgrundlage für die Privatisierung dar, da es durch Art. 25 des Einigungsvertrags im wesentlichen aufrechterhalten blieb. Mit Wirkung vom 1. 7. 1990 wurden kraft Gesetzes alle Kombinate in A Gs und alle Kombinatsbetriebe sowie sonstigen Einrichtungen in $\mathrm{GmbHs}$ umgewandelt. Alleingesellschafter wurde die Treuhandanstalt, eine rechtsfähige Anstalt des öffentlichen Rechts, die seit dem Einigungsvertrag der Aufsicht des Bundesministers der Finanzen unterliegt. Im Laufe des Monats Juli 1990 konnten die bisherigen Betriebsleiter abberufen werden, doch wurde davon nur in Ausnahmefällen Gebrauch gemacht.

In $₫$ r r Abs. 2 Satz 2 Treuhandgesetz wird eine sehr grundsätzliche Aussage getroffen: "Die Umwandlung bewirkt gleichzeitig den Ưbergang des Vermögens aus der

38 Näher Däubler AuR 1991, 197 f.

39 Einzelheiten bei Däubler, Ratgeber Arbeitsrecht, S. 413.

$40 \mathrm{Zu}$ diesem auf Lenin zurückgehenden Grundsatz s. Deppe, in; Jung-Dcppe u. a., BRD-DDR. Vergleich der Gesellschafissysteme, Köln 1971, S. $121 \mathrm{ff}$.

$41 \mathrm{GBI} . \mathrm{I}, 107=\mathrm{NJW} 1990,1286$.

42 Dornberger-Dornberger DB 1990, 3042; Lachmann DtZ 1990, 239

43 GBI. I, 300. Einzelheiten bei Horn, Das Zivil- und Wirtschaftsrecht im neuen Bundesgebiet, Koln 1991, S. $318 \mathrm{f}$. 
Fondsinhaberschaft der bisherigen Wirtschaftseinheit sowie des in Rechtsträgerschaft befindlichen Grund und Bodens in das Eigentum der Kapitalgesellschaft «. Die Verwandlung des Nutzungsrechts in Privateigentum verstieß gegen die Unantastbarkeit des Volkseigentums; gleichzeitig beschloß daher das Parlament ein Gesetz zur Änderung und Ergänzung der Verfassung der DDR ${ }^{44}$, das in Art. 2 Satz. I ausdrücklich das Privateigentum an Grund und Boden sowie an Produktionsmitteln gewährleistete.

Nach Art. 25 Abs. $x$ Satz $x$ des Einigungsvertrages ist die Treuhandanstalt damit beauftragt, "gemäß den Bestimmungen des Treuhandgesetzes « die früheren volkseigenen Betriebe "wettbewerblich zu strukturieren und zu privatisieren «. In der Praxis ergeben sich hier große Schwierigkeiten. Bis Ende September 1991 waren von den mehr als 8000 Kombinaten und VEBs 3788 "Unternehmen und Betriebsteile« an Private veräußert"s - eine recht optimistisch gefärbte Statistik, da größere Unternehmen oft in $\mathrm{r} 5-20$ "Betriebsteile " aufgespalten und an unterschiedliche Interessenten veräußert werden. ${ }^{6}$ Auch bestreitet niemand, daß die "Filetstücke und Rosinen" sich leicht verkaufen ließen, während Einheiten mit großem Investitionsbedarf nur mit großer Mühe (und hohen Subventionen) einen Käufer finden. ${ }^{46 .}$

\section{b) Rückgabe des nach 1949 enteigneten Vermögens}

Nach dem durch den Einigungsvertrag geschaffenen "Gesetz zur Regelung offener Vermögensfragen ${ }^{47}$ sind im Prinzip alle Vermögenswerte den früheren Inhabern zurückzuerstatten, die nach 1949 in Volkseigentum überführt wurden. Dies gilt insbesondere für Klein- und Mittelbetriebe, die 1972 vom Staat übernommen wurden. $4^{8}$ Der größte Teil des Volkseigentums war allerdings aufgrund von Enteignungen entstanden, die die sowjetische Besatzungsmacht bereits vor $x 949$ vorgenommen hatte. An dem dadurch geschaffenen Zustand sollte nichts mehr geändert werden; die entsprechenden Bestinumungen des Einigungsvertrags wurden vom Bundesverfassungsgericht bestätigt. ${ }^{49}$ Wer einen nicht völlig aussichtslosen Antrag auf Rückerstattung z. B. eines Grundstücks stellt, kann im Regelfall verhindern, daß der derzeit Verfügungsberechtigte irgendwelche Veränderungen vornimmt..$^{\circ}$ Investoren können allerdings unter bestimmten Voraussetzungen eine sog. Investitionsbescheinigung erhalten, die den Rückgabeanspruch ausschließt und den früheren Eigentümer auf eine Geldentschädigung beschränkt. Dasselbe gilt, wenn Eigentum oder andere Rechte an Grundstücken gutgläubig erworben wurden oder wenn die Rückgabe nur um den Preis der Zerschlagung eines Unternehmens oder einer einheitlichen Siedlungsmaßnahme möglich wäre.

44 GBI. I, 299 (vom 22.6. 1990).

4 Rühl WSI-Mitt. 1991,682

46 Beispiel: AWE Eisenach ("Wartburg $\alpha$ ), die als solche liquidier werden, auf deren Gelände sich aber über 20 Investoren angesiedelt haben, die größtenteils Betriebsteile (und ein Fünftel der ursprünglichen Belegschaft) übernommen haben.

46a Zur Bindung der Treuhand an verfassungsrechtliche Prinzipien, insbes. an das Gebor zur Vermeidung von Arbeitslosigkeit, s. Blechmann, Zur verfassungsrechtlichen Sanierungspflicht der Treuhandanstalt, Köln u. a. 1992

47 Anlage II Kap. III Sachgebiet B Abschnitt I Nr.s zum Einigungsvertrag.

$4^{8} \mathrm{Zu}$ den Finzelheiten, die hier nicht dargestellt werden können, s. insbes. Jung-Vec JuS 1991, 714 ff.; Horn (Fn. 43), S. $221 \mathrm{ff}$

49 BVerfG EuGRZ 199 I, 12 iff. Insoweit greift aber der unter a dargestellte allgemeine Privatisierungsauftrag der Treuhand ein.

so Bestätigt durch BVerfG EuGRZ 1991, 291 ff. 
Das von der SED und den mit ihr verbündeten sog. Blockparteien erworbene Ver-

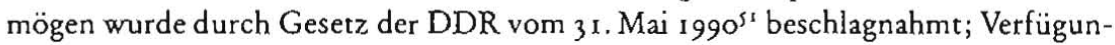
gen über einzelne Gegenstände sind nur mit Zustimmung der Treuhandanstalt möglich. Maßgebender Stichtag ist der 7. Oktober 1989 . Eine Rückgabe ist nur insoweit vorgesehen, als die Partei beweisen kann, die entsprechenden Gegenstände »nach materiell-rechtsstaatlichen Grundsätzen « erworben zu haben. Mit Rücksicht darauf, daß nach dem Stichtag geleistete Mitgliedsbeiträge nicht von der Beschlagnahme erfaßt werden, hat das Bundesverfassungsgericht keinen Verstoß gegen den verfassungsrechtlich garantierten Parteienstatus nach Art. 2 I Abs. I GG erkennen können. ${ }^{52}$ Die Tatsache, daß Eigentum gesellschaftlicher Organisationen nach der am 31. Mai 1990 noch geltenden DDR-Verfassung nicht entzogen werden konnte, hat bislang keine Rolle gespielt.

\section{Die Säuberung des Staatsapparates}

Die Übernahme der staatlichen Verwaltung der ehemaligen DDR warf aus bundesrepublikanischer Sicht zwei Hauptprobleme auf. Zum einen fielen bestimmte Aufgaben wie z. B. die des Außenministeriums oder die des Amtes für Preise weg. Zum andern entsprach die personelle Besetzung vieler Funktionen nicht den Vorstellungen der Bundesregierung: Die Anzahl der für bestimmte Arbeitsaufgaben eingesetzten Beschäftigten war sehr viel größer als in der (alten) Bundesrepublik, an vielen Stellen, insbesondere in Leitungsfunktionen, saßen Personen, die ein besonderes $\mathrm{Maß}$ an Loyalität gegenüber dem bisherigen Gesellschaftssystem entwickelt hatten. Beidem versuchte Kap. XIX der Anlage I zum Einigungsvertrag Rechnung zu tragen.

\section{a) Die Abwicklung einzelner Einrichtungen}

Bis 3. r. r991 konnten der Bund bzw. die (neugeschaffenen) Länder darüber entscheiden, welche Einrichtungen oder welche Teile von Einrichtungen sie übernehmen wollten. Eine negative Entscheidung hatte zur Folge, daß die dort beschäftigten Arbeitnehmer in die sog. Warteschleife geschickt wurden. Vom Zeitpunkt der Entscheidung an ruhte das Arbeitsverhältnis, nach 6 Monaten lief es automatisch aus. Wer am 3. 10. 1990 bereits das 50. Lebensjahr vollendet hatte, erhielt eine "Übergangsfrist « von 9 Monaten. Während dieser Zeit wurde ein sog. Wartegeld bezahlt, das in etwa dem Arbeitslosengeld entsprach. Außerdem war der Arbeitgeber gehalten, Weiterbildungsangebote zu machen und sich um eine anderweitige Beschäftigungsmöglichkeit zu bemühen - weshalb man von "Warteschleife" sprach, obwohl sich in der Praxis nur sehr selten Möglichkeiten zur Wiedereingliederung ergaben.

Die Suspendierung und anschließende Auflösung der Arbeitsverhältnisse tritt nach dem Text des Einigungsvertrages auch dann ein, wenn es sich um besonders geschützte Arbeitnehmergruppen wie werdende Mütter, Schwerbehinderte oder Personalratsmitglieder handelt. In der Praxis wurde überdies vom Mittel der "Abwicklung « in der Weise Gebrauch gemacht, daß zwar die Einrichtung als solche erhalten blieb, die Hälfte des Personals jedoch »in die Warteschleife geschickt « wurde. Beides

\$1 GBI. I, 275.

s2 BVerfG EuGRZ 1991, $287 \mathrm{ff}$. 
führte zu einem Verfahren vor dem Bundesverfassungsgericht, das innerhalb weniger

Monate eine Entscheidung traf. Danach läßt sich die getroffene Regelung zwar bei verfassungskonformer Auslegung mit dem Grundgesetz vereinbaren, doch gibt es zwei wichtige Modifikationen: 53 Die Suspendierung und Auflösung der Arbeitsverhältnisse ergreift nicht Arbeitnehmerinnen unter Mutterschutz, da dies gegen Art. 6 Abs. 4 GG verstoßen würde. Außerdem muß sog. Problemgruppen - dazu zählt das Verfassungsgericht Schwerbehinderte, ältere Arbeitnehmer und Alleinerziehende eine »begründete Aussicht" auf eine neue Stelle im öffentlichen Dienst geboten werden. Schließlich darf das Mittel der "Abwicklung " nicht zu Zwecken der "Personalausdünnung " verwendet werden; insoweit liegt ein Mißbrauch vor, gegen den sich die Betroffenen vor den zuständigen Gerichten zur Wehr setzen können. ${ }^{34}$ In der Entscheidung zur Abwicklung der Akademie der Wissenschaften der DDR hat das BVerfG diese Grundsätze bestätigt und zusätzlich verlangt, denjenigen, die sich bei einer "Nachfolgeorganisation« beworben hatten, eine Mindestauflösungsfrist von einem Monat zum Monatsende einzuräumen, damit sie sich nach neuen Arbeitsmöglichkeiten umsehen können. ${ }^{5}$ Die Anwendung dieser Grundsätze hat zahlreiche Interpretationskontroversen ausgelöst $;^{56}$ dennoch konnten einige Mißbrauchsfälle gerichtlich korrigiert werden."7

\section{b) Sonderkündigungsgründe}

Auch wer in einem von der Bundesrepublik oder einem Land übernommenen Teil der Verwaltung tätig ist, kann sich seines Arbeitsplatzes nicht sicher sein. Abs. 4 der einschlägigen Regelung im Einigungsvertrags8 räumt dem öffentlichen Arbeitgeber zusätzliche Möglichkeiten zur ordentlichen, Abs. s solche zur außerordentlichen Kündigung ein.

Nach Abs. 4 Ziff. $x$ ist die ordentliche Kündigung eines Arbeitsverhälnnisses in der öffentlichen Verwaltung "auch zulässig, wenn der Arbeitnehmer wegen mangelnder fachlicher Qualifikation oder persönlicher Eignung den Anforderungen nicht entspricht«. Fehlende persönliche Eignung wird insbesondere dann angenommen, wenn sich jemand in der Vergangenheit politisch exponiert hat. Alle übernommenen Beschäftigten mußten deshalb eingehende Fragebögen ausfüllen, die sich insbesondere auf Funktionen in Parteien und Gewerkschaften sowie auf den Erhalt von Auszeichnungen bezogen. Ob ggf. gekündigt wird, ist eine Ermessensentscheidung der zuständigen Verwaltung. Im Lande Berlin existieren Richtlinien, wonach eine Kündigung immer dann vorgenommen wird, wenn der Betroffene Leiter der Abteilungsparteiorganisation (APO) der SED war, also an der Spitze der kleinsten Einheit der Partei stand. Funktionen auf Kreis- oder gar Bezirksebene reichen erst recht aus. ${ }^{99} \mathrm{Ob}$ diese Regelung mit dem Grundgesetz und dem ILO-Übereinkommen Nr. I I vereinbar ist, erscheint sehr $z$ weifelhaft ${ }^{60}$; eine höchstrichterliche Klärung ist

53 BVerfG EuGRZ 1991, 133 ff.

\$4 Weitere Einzelheiten bei Däubler PersR 1991, I93 ff.; Schmalz PersR 1991, Is 3 ff.; Wolter ZTR r991, ${ }_{273} \mathrm{ff}$.; Zundel ZTR 1991, $311 \mathrm{ff}$. Dic Prozeßgeschichte ist dokumentiert bei Wulf-Mathies (Hrsg.), - Warteschleife und Einigungsvertrag, Köln 1992.

ss BVerfG EuGRZ 1992, 110. Wer enva am 15. 12.1991 eine Ablehnung erhielt, mußte nicht nur bis 31. 12. 1991 (so Art. 38 Abs. 3 Satz I Einigungsvertrag), sondern bis 31. 1. 1992 weiterbeschaftigt werden.

s6 Überblick bei Fenski-Linck NZA 1992, $349 \mathrm{f}$.

57 S. etwa OVG Berlin ZTR r991, 338 (Humboldt-Universitat Berlin).

58 Kap. XIX Sachgebiet A Abschnitt III Nr. I der Anlage I zum Einigungsvertrag.

s9 Rundschreiben II Nr. 82/1990 der Senatsverwaltung für Inneres/Magistratsvenvalung fur Inneres vom 4. 12. 1990 .

$60 \mathrm{Vgl}$. zu den (noch sehr viel weitergehenden) Vorschlägen der Bundesregierung bei den Verbandlungen 
bislang nicht erfolgt. ${ }^{61}$ Daneben spielen die anderen Zusatzgründe für eine ordentliche Kündigung nur eine geringere Rolle; so kann der einzelne "wegen mangelnden Bedarfs « oder deshalb gekündigt werden, weil seine Beschäftigungsstelle aufgelöst wird - beides würde meist auch nach allgemeinem Kündigungsschutzrecht ausreichen. ${ }^{62}$

Nach Abs. s der einschlägigen Regelung ${ }^{\sigma_{3}}$ ist ein wichtiger Grund für eine fristlose Kündigung »insbesondere dann gegeben, wenn der Arbeitnehmer gegen die Grundsätze der Menschlichkeit oder Rechtsstaatlichkeit verstoßen hat (Ziff. I) oder für das frühere Ministerium für Staatssicherheit tätig war (Ziff. 2) und deshalb ein Festhalten am Arbeitsverhältnis unzumutbar erscheint. Nach der amtlichen Begründung soll diese Vorschrift die Trennung von vorbelastetem Personal erleichtern und insbesondere »ein politisches Signal setzen ${ }^{64}$.

Ziffer $\mathrm{x}$ hat bislang wohl keine praktische Bedeutung erlangt; jedenfalls sind Gerichtsentscheidungen zu dieser Vorschrift nicht ersichtlich. Die amtliche Begründung schwächt ihren Wortlaut dadurch etwas ab, daß sie einen Verstoß gegen »elementare Grundsätze « der Menschlichkeit und Rechtsstaatlichkeit voraussetzt. ${ }^{6 s}$ Zur näheren Präzisierung wird im Text selbst auf den Internationalen Pakt über bürgerliche und politische Rechte vom 19. Dezember 1966 sowie auf die Allgemeine Erklärung der Menschenrechte vom Io. Dezember 1948 verwiesen. ${ }^{66}$ Die letztere ist damit zum ersten Mal in das geltende Recht rezipiert worden, da sie ja nicht einmal völkerrechtliche Verbindlichkeit beanprucht. Was die Ziff. 2 betrifft, so reicht nach der amtlichen Begründung auch eine nebenamtliche Tätigkeit aus; ${ }^{67}$ um festzustellen, ob jemand Mitarbeiter des besagten Ministeriums war, dürfen dessen Dateien benutzt werden. Die Rechtsprechung zu den „Stasi-Kündigungen « ist recht umfangreich; erfolgreiche Kündigungsschutzklagen sind bisher die absolute Ausnahme. ${ }^{68}$ Auch wer als Tischler bei der Bundespost arbeitet, kann nach Auffassung des LAG Berlin fristlos gekündigt werden, wenn er zuvor lange Jahre bei der Funkabwehr des MfS tätig war. ${ }^{69}$ Die im Einigungsvertrag vorgesehene Einzelfallprüfung kann dazu führen, daß die geringe Bedeutung der früheren Stasi-Funktion die Weiterarbeit zumurbar macht. ${ }^{70}$

Nach fristloser Kündigung wird gcmäß $\$ \$$ I19, I I9a AFG zwölf Wochen lang kein Arbeitslosengeld bezahlt.

\section{Die Kürzung von Intelligenzler-Renten*}

In der DDR gab es eine Reihe sog. Zusatzversorgungssysteme, die insbesondere Personen in Leitungsfunktionen, aber z. B. auch Lehrern und Ärzten eine zusätzli-

über den Einigungsvertrag die kritische Analyse durch Lörcher PersR 1990, 217 ff. Zum Bericht des ILO-Untersuchungsausschusses über die parallel licgende Praxis der Berufsverbote s. Dammann-Siemantel (Hrsg.), Berufsverbote und Menschenrechte in der Bundesrepublik Deurschland - Report der Internationalen Arbeitsorganısation, Köln 1987 ; Bobke AiB 1988, 234.

6I Das ArbG Berlin (DB 1992, 586) will die Grundstitze über die Behandlung von „Verfassungsfeinden « im öffentlichen Dienst entsprechend anwenden und den Betroffenen eine "Neuorientierung emö̈glichen.

62 Näher Preis PersR 1991, 201 ff. und Fenski-Linck NZA 1992, 339.

63 S. Fn 58.

64 BT-Drucksache $11 / 78$ I 7 S. 180 li.Sp.

65 Ebd.

66 Beide Texte sind auszugsweise abgedruckt bei Däubler-Kittner-Lörcher (Hrsg.), Internationale Arbeitsund Sozialordnung, Koln 1990, uncer Nr. 110 bzw. 120.

67 BT-Drucksache $11 / 7817$ S. 180.

68 Überblick über erst- und zweitinstanzliche Entscheidungen bei Korinth NZA 1992, 354. S. weiter U. Scholz BB $\times 991,2515 \mathrm{ff}$. Schr restrikriv nunmehr BAG DB $\times 992,1352$.

69 LAG Berlin BB 1992, 215

70 LAG Berlin BB 1992,713 : Kuchenhilfe. 
che Altersversorgung garantierten. Dies hing damit zusammen, daß in der gesetzlichen Rentenversicherung die sog. Beitragsbemessungsgrenze bei 600,-DM lag; das darüber hinausgehende Einkommen blieb bei der Rentenberechnung unberücksichtigt $^{7 t}$. Der Einigungsvertrag ${ }^{72}$ sah die Schließung dieser Versorgungssysteme und die Überführung der Anwartschaften und Ansprüche in die allgemeine Rentenversicherung vor; die dabei notwendig werdende Neuberechnung durfte den für Juli 1990 geschuldeten Betrag nicht unterschreiten. Das Rentenüberleitungsgesetz vom 25. Juli $199 \mathrm{r}^{73}$ ist von diesen Vorgaben zu Lasten der Rentner und Anwartschaftsberechtigten abgewichen: Als Berechnungsgrundlage wurde für die Vergangenheit das Durchschnittseinkommen aller DDR-Bürger festgelegt, außerdem darf ein Gesamtbetrag von monatlich $2050,-\mathrm{DM}$ nicht überschritten werden..$^{74}$ In der öffentlichen Anhörung des Bundestagsausschusses für Arbeit und Sozialordnung waren allerdings verfassungsrechtliche Bedenken geäußert worden7s; es sei nicht Aufgabe der Sozialversicherung, die politische Vergangenheit zu bewältigen. Auch wurde darauf verwiesen, daß nach 1945 derartige Sanktionen nicht einmal gegen Angehörige der Gestapo verhängt wurden. Voraussichtlich wird auch hier das Verfassungsgericht zu entscheiden haben.

\section{Die Implementation des neuen Rechts}

\section{Grundsätzliche Akzeptanzprobleme?}

Legt man Erfahrungen anderer Länder zugrunde, muß man vermuten, daß ein derart massiver Rechtsexport bei der betroffenen Bevölkerung auf Widerstand stößt. Erfahrungen etwa in Japan ${ }^{76}$ oder in der Türkei77, die sich auf einen sehr viel bescheideneren "Rechtsimport " bezogen, machen deutlich, daß sich das neue Recht erst im Laufe einer längeren Entwicklung durchsetzte und dabei einem erheblichen Assimilationsdruck ausgesetzt war.

Die Situation in der früheren DDR ist eine grundsätzlich andere: Die bisherige Ordnung und ihr Recht sind so diskreditiert, daß sich nur kleine Minderheiten zu seiner argumentativen Verteidigung bereitfinden. Dem bundesdeutschen Recht wird im Grunde nichts entgegengesetzt; es trifft auf ein politisches Vakuum, seine Legitimität wird nicht angezweifelt.

Die Ursachen für die Diskreditierung können hier nur skizziert werden. ${ }^{8}$ Politisch war es die Allgegenwart des Staates, die geschriebene und ungeschriebene Reglementierung des Alltagslebens, die Distanz und Widerstand bei der Bevölkerung schuf. Ökonomisch wuchsen in den 8oer Jahren die wirtschaftlichen Schwierigkeiten, ohne daß ein Ausweg auch nur angedeutet worden wäre. Das Gesellschaftssystem war als

\footnotetext{
71 Bei anderen Arbeitnehmern griff die Frciwillige Zusatzrentenversicherung (FZR) ein, die jedoch insgesamt weniger günstig war.

72 Kap. VIII Sachgubiet H Abschnitt UII Nr. 9 der Anlage II.

73 BGBI. I, 1606.

74 Einzelheiten bei Michaelis ZTR r991, 403; Nilges AuA 1991, 332; Rahn DiZ, 1992, s.

75 Anhörung vom 16. 5. 1991, Stellungnahmen von Azzola und Isensee.

76 Vgl. etwa Gould, Japan's Reshaping of American Labor Law, Cambridge/London 1984.

77 Ernst Hirsch, Rezeption als Sozialer Prozeß. Erläutert am Beispıel der Türkei, Berlin 198 1 (mit Bezug auf Zivil- und Handelsrecht).

78 Eingehender Däubler GMH 1990, 353 ff.; aufschlußreich insbes. Staritz, Geschichte der DDR 1949-1985, Frankfurt/Main 1985, sowie Blanke-Erd (Hrsg.), DDR. Ein Staat vergeht, Frankfurt/Main 
solches nicht "lernfähig «; anders als Kaiser und Könige tolerierte das Politbüro nicht einmal einen Hofnarren, der bisweilen in vielleicht überspitzter Form die Wahrheit sagte ${ }^{79}$. Die zentralistischen Steuerungsmechanismen - bewußt oder unbewußt dem Industriebetrieb des 19 . Jahrhunderts entlehnt - waren ungeeignet für ein so komplexes System wie eine moderne Industriegesellschaft ${ }^{80}$. Dazu kam die internationale Lage mit der immer sichtbarer werdenden Krise der übrigen sozialistischen Länder und dem prosperierenden Alternativmodell Bundesrepublik vor der eigenen Haustüre. Die überwältigende Mehrheit der Bevölkerung wollte unter diesen Umständen die Wiedervereinigung und bejahte die politische und wirtschaftliche Ordnung der Bundesrepublik. Daß dabei bisweilen die (ja auch in der DDR wahrnehmbare) Fernsehwelt mit der Realität in eins gesetzt wurde, spielte letztlich keine Rolle: ex occidente lux. ${ }^{81}$

\section{Die Bildung von Gewerkschaften und Arbeitgeberverbänden}

Arbeitsrecht ist noch lange kein »lebendiges Recht «, wenn es lediglich auf keinen Widerstand stößt. Notwendig ist, daß sich Akteure bilden, die von der Tarifautonomie Gebrauch machen, die die oft bescheidenen oder ausfüllungsbedürftigen gesetzlichen Normen durch einen besseren tariflichen Schutzstandard ergänzen.

Die äußeren Abläufe sind schnell skizziert. Der FDGB sowie die einzelnen Branchengewerkschaften beschlossen im Laufe des Jahres 1990 ihre Auflösung: Auch wenn einzelne Personen weiterhin das Vertrauen der Belegschaften besaßen, fand die Organisation als solche keine Akzeptanz mehr. Die westdeutschen Gewerkschaften rekrutierten Mitglieder in den neuen Bundesländern; in den Auflösungsbeschlüssen der DDR-Gewerkschaften wurde meist ein Übertritt empfohlen. Trotz des beträchtlichen Vermögens des FDGB verzichtete der DGB auf eine Fusion, da dies den unerwünschten Anschein einer Kontinuität erweckt hätte. Die Mitgliederzahlen der DGB-Gewerkschaften gingen steil in die Höhe; obwohl derzeit noch keine verläßlichen Zahlenangaben verfügbar sind, deuten alle Anzeichen darauf hin, daß der Organisationsgrad erheblich höher als in der alten Bundesrepublik liegt. ${ }^{82}$ Dies dürfte mit den großen sozialen Problemen, aber auch damit zusammenhängen, daß sich die Bevölkerung der früheren DDR häufig gegenüber den alles besser wissenden Westlern deklassiert fühlt und die Gewerkschaft als eine Organisation empfinder, wo man als Gleicher akzeptiert wird und überdies etwas für die eigenen Interessen tun kann. Die DAG und der Deutsche Beamtenbund haben in den neuen Bundesländern gleichfalls Mitglieder geworben; zahlenmäßig bleiben sie genau wie in der alten Bundesrepublik weit hinter den DGB-Gewerkschaften zurück. Auf Arbeitgeberseite ergeben sich insoweit gewisse Abweichungen, als Privatunternehmen oft nicht dem zuständigen Arbeitgeberverband angehören und deshalb sehr viel mehr Firmen-

79 In modernen Industriegesellschaften wird diesc Funktion häufig von Wissenschaftern wahrgenommen.

8o Blanke, in: Blanke/Erd (Fn. 78 ), S. 193 .

81 Ob das sozialistische Recht von vomherein als oktroiert empfunden wurde und deshalb nur gcringe Verankerung besaß, läßt sich nicht sicher beurteilen. Zur Kategorie der „forced adaptation * am Beispiel Ungarns s. Kulcsár, Forced adaptation and Law-Making: A Funcrional Aspcct of Comparative Law, in: Institute of Comparative Law, Waseda-University, Recht in Ost und West, Festschrift zum dreißigjährigen Jubiläum des Instituts für Rechtsvergleichung der Waseda-Universität, Tokio 1988, S. 245 , 259.

82 In der alten Bundesrepublik organisieren die DGB-Gewerkschaften 31,8\% aller Beschäftigten; dazu kommen ca. 2,5\% Mitglieder der DAG und des DBB. Nähere Angaben bei Schmitz-Tiemann-Löhrlein, in: Kitıner (Hrsg.), Gewerkschaftsjahrbuch 1991, Köln 1991, S. 81 ff. Neueste Angaben in: AuR 1992, 211 ; der Zuwachs von 3,86 Mio Mitglieder 1991 verweist auf einen Organisationsgrad von gut $50 \%$ in den neuen Bundeslandern, konstante Zahlen in den alten vorausgesetze. 
tarife als im Westen abgeschlossen werden müssen. ${ }^{83}$ Z wischen den Arbeitgeberver-

bänden und den Gewerkschaften besteht jedoch Einverständnis, am System der

Flächentarife festzuhalten; aufs Ganze gesehen halten sich die Abweichungen vom westlichen Modell daher in Grenzen.

Die Übernahme der bundesdeutschen Gewerkschaftsstrukturen war - abstrakt gesehen - keine Selbstverständlichkeit. ${ }^{83 \mathrm{a}}$ Ähnlich wie in Ungarn oder Polen hätte man sich auch ein Nebeneinander verschiedener Richtungsgewerkschaften vorstellen können. Auch wäre es plausibel gewesen, wenn sich in zahlreichen Betrieben autonome Interessenvertretungen gebildet hätten, die angesichts bisheriger Erfahrungen jede »Reglementierung " durch eine Großorganisation abgelehnt hätten. Beides passierte nicht. Richtungsgewerkschaften wären nur dann in Betracht gekommen, wenn sich in den Betrieben kirchliche, sozialdemokratische, anarchistische usw. Gruppen gebildet und aktiv in den Prozeß politischer Umwandlung eingeschaltet hätten. Diese Voraussetzung fehlte; die "Wende « kam ohne größere betriebliche Aktionen zustande. Die zweite potentielle Entwicklungsvariante scheiterte an der Schwäche der betrieblichen Interessenvertretungen: Konfrontiert mit der neuen Ordnung, die selbst den Alltag revolutionierte ${ }^{\beta_{4}}$, waren sie froh, von erfahrenen Gewerkschaftern aus dem Westen ein wenig lernen zu können. Dazu kam das Prestige alles "Westlichen«, von dem auch die Gewerkschaften profitierten ${ }^{8}$. Schließlich gab cs niemanden im Westen, der die Expansion der DGB-Gewerkschaften ernsthaft behindert hätte; den Regeln der Sozialpartnerschaft entsprach es, den Arbeitnehmerorganisationen ihren Anteil am zu verteilenden Kuchen zu überlassen.

Die organisatorischen Erfolge dürfen nicht darüber hinwegtäuschen, daß die deutschen Gewerkschaften nunmehr eine sehr heterogene Mitgliedschaft repräsentieren: Unterschiedlich sind nicht nur Lebensniveau und Löhne ${ }^{86}$, sondern auch die Mentalitäten; während sich im Westen langsam eine Öffnung hin zu Fragen des Umweltschutzes und des Lebens außerhalb der Fabrik vollzieht, herrscht im Osten noch immer die rein arbeitsbezogene Perspektive vor.

\section{Bildung von Betriebsräten}

Nach ẹiner ausdrücklichen Bestimmung im Einigungsvertrag ${ }^{87}$ mußten bis zum 30. Juni 199x Betriebsratswahlen stattfinden. Um eine vertretungslose Zeit zu vermeiden, wurden diejenigen betrieblichen Interessenvertretungen wie Betriebsräte behandelt, die bis zum 31 . Oktober 1990 von der Mehrheit der Belegschaft in geheimer Abstimmung nach demokratischen Grundsätzen gewählt worden waren. ${ }^{88}$ Dies konnten im Einzelfall auch Betriebsgewerkschaftsleitungen nach altem Recht sein; im Regelfall waren allerdings nach dem politischen Umbruch im Oktober 1989 in einem formlosen Verfahren neue Interessenvertretungen bestimmt worden. Soweit ersichtlich, sind zumindest in den mittleren und größeren Betrieben Betriebsräte

83 Bispinck/WSI-Tarifarchiv WSI-Mitt. 1992, 122

83 a Adelheid Hege, Un Syndicalisme unique pour deux Systèmes sociaux? Enjeux et Perspectives de l'Unification syndicale en Allenagne, Beitrag zum Seminar des Europaischen Hochschulinstituts in Florenz vom 30. bis 31. Mai 1991, Manuskript, S. 2.

84 S. die eindrückliche Schilderung bei Baroth GMH r991, 4c9 ff.

85 Dies ging so seit, daß man vernünftige Organisationsprinzipien wie die in der DDR praktızierte Zusammenfassung der Beschäfrigten von Handel, Nahrungsgewerbe und Gaststatten in einer Dienstleistungsyc'werkschaft wieder aufgab - dazu Bunke GMH r $991,437$.

86 Zur Tarifpolitiks. unten $\mathrm{V}_{2}$

87 Kap. VIII Sachgebiet A Abschnitt III Nr. 12 b der Anlage I

88 Kap. VIII Sachgelsiet A Abschnit IU Nr. 4 der Anlage II zum Einigungsvertrag, wonach die DDR-VO vom 11. Juli 1990 (GBI. I, 715) aufrechterhalten blieb. 
gewählt worden. Obwohl noch keine ausreichenden Zahlen vorliegen, besteht die verbreitete Einschätzung, daß nicht weniger Betriebsräte als in vergleichbaren $\mathrm{Be}$ trieben der alten Bundesrepublik existieren.

\section{Schwierigkeiten der Akteure mit dem neuen Recht}

Die Existenz von Gewerkschaften, Arbeitgeberverbänden und Betriebsräten ist eine notwendige, aber keine hinreichende Bedingung für wirksame Interessenvertretung und Entwicklung angemessener Schutzstandards. Hinzukommen muß die Fähigkeit, zu verhandeln und vorhandene Rechte effektiv durchzusetzen. Insoweit bestehen zwei wesentliche Schwierigkeiten.

Das neue Recht ist ungleich komplexer als das alte. Die Tatsache, daß an die Stelle einer relativ übersichtlichen Kodifikation eine Vielzahl von Einzelgesetzen tritt, ist dabei das geringere Problem. Viel wichtiger ist das feine Netz von richterrechtlichen Normen und Kommentarmeinungen, das im Laufe von 40 Jahren entwickelt wurde. Wer damit keine Erfahrungen hat, wird sich wie in einem undurchdringlichen Dikkicht fühlen. Die Unsicherheit beim Umgang mit dem Neuen ist auch auf Arbeitgeberseite groß. Zwar gibt es mittlerweile eine recht umfangreiche Einführungsliteratur ${ }^{89}$, doch kann sie die Informationsdefizite und die mangelnde Erfahrung nicht kurzfristig ausgleichen.

Die zweite Schwierigkeit liegt in eingefahrenen Verhaltensweisen. Die Fähigkeit, nein zu sagen und eigene Interessen in der Öffentlichkeit ebenso wie in einer Nachtsitzung der Einigungsstelle durchzusetzen, wurde in einem Staat nicht gelemt, wo das "Ausscheren « als wunderliches oder bösartiges Verhalten galt. Allgemeine kulturelle Standards wie Rücksichtnahme und Bescheidenheit machen den Schritt zum selbstbewußten, auf gute Selbstdarstellung bedachten Interessenvertreter noch um einiges größer.9 ${ }^{\circ}$

\section{Funktionsdefizite bei den Gerichten}

Die größten Probleme existieren im Bereich des gerichtlichen Rechtsschutzes. Die Verfahrensdauer liegt weit höher als in den alten Bundesländern; oft muß man ein halbes oder gar ein ganzes Jahr warten, bis man einen Gütetermin erhält, bei dem lediglich Vergleichsverhandlungen geführt werden. Die Gründe haben eine quantitative wie eine qualitative Dimension.

Die Zahl der Richter lag in der ehemaligen DDR drastisch niedriger als in der Bundesrepublik. Während letztere im Jahre 1989 über 17627 Richter verfügte ${ }^{91}$, übten in der DDR nur ${ }_{4} 493$ Personen diese Funktion aus ${ }^{92}$. Berücksichtigt man die unterschiedliche Bevölkerungszahl (3,7:1), so war die „Richterdichte" in der alten Bundesrepublik gleichwohl un den Faktor 3,2 größer. Sehr unterschiedlich war auch die Inanspruchnahme der Gerichte: 360000 neu anhängig gemachten arbeits-

89 Neben dem in Fn. 16 genannten Buch ist insbes. zu verweisen auf Aufhauser-Bobke-Warga, Einführung in das Arbeits- und Sozialrecht der Bundesrepublik Deutschland, München 1990; Böckel-Leier-Thiel, Arbeitsrecht. Wie schützt es mich? Ratgeber für die neuen Bundesländer, Hamburg-Berlin-Dresden 1991 ; Viechen-Schwedes, Arbeitsrecht 1990/91 in der betrieblichen Praxis. Mit den Sonderregelungen in den neuen Bundeslandern, München 1990; Walker, Das Arbeitsrecht in den neuen Bundeslandern, München 1991 .

90 Áhnliche Einschäczungen bei Biedenkopf GMH 1991, 274 f.; Bunke GMH 1991, 473. Jurgen Kuczynski berichtet in seinem Tagebuch, das haufigste Prädikat, mit dem Schuldirektoren ihre Absolventen den Universitäten empfahlen, sei n Beseheidenheit und Zurückhaltung" gewesen (J. Kuczynski, Schwierige Jahre - Mit einem besseren Ende? Tagebuchblätter 1987-1989, Berlin 1990, S. 213).

91 Statistisches Jahrbuch für die Bundesrepublik Deutschland 1990, 336.

92 Statistisches Jahrbuch der DDR 1990, 448. 
gerichtlichen Verfahren in der alten Bundesrepublik ${ }^{93}$ standen im Jahre 1989 ganze Is 449 in der damaligen DDR gegenüber; ${ }^{94}$ unter Berücksichtigung der Bevölkerungszahl entspricht dies einem Verhältnis von $6,3: 1$. Die Situation ändert sich allerdings, wenn man die knapp 60000 Verfahren hinzurechnet, die vor den Konfliktkommissionen anhängig wurden. ${ }^{95}$ In diesem Fall reduziert sich das Verhältnis auf $1,34: 1$, läßt also die Differenzen sehr viel geringer erscheinen. In der Gegenwart ist dies allerdings fast ohne Bedeutung, da die Schiedsstellen ${ }^{96}$ nur teilweise eingerichtet sind und da sie in der Regel keine definitive Streitschlichtung zustande bringen. Angesichts der Massenentlassungen kommt auf die wenigen Richter eine Prozeßflut zu, der sie nicht Herr werden können.

Die zweite, qualitative Seite ist möglicherweise noch gravierender. Alle DDR-Richter müssen sich einer fachlichen und politischen Überprüfung unterziehen, die entweder von der Regierung oder einem parlamentarisch gewählten Gremium vorgenommen wird. Dies schuf während eines Zeitraums von rund $\mathrm{x} x / 2$ Jahren ein $\mathrm{Maß}$ von existentieller Unsicherheit, das bis in die Urteilstätigkeit hincinwirkte. ${ }^{97}$ Viele Richter, die in politischen Strafverfahren mitgewirkt hatten, gaben ihr Amt freiwillig auf und ließen sich als Rechtsanwälte nieder. Aus den westlichen Bundesländern entsandte Richter konnten zwar diese Lücken einigermaßen füllen, schufen jedoch zwei Gruppen von Richtern: Den blutigen Anfängern standen von nun an die erfahrenen Meister gegenüber.

Die lange Dauer der Verfahren wirkt auf die Arbeitsverhältnisse und das Verhalten der kollektiven Akteure zurück: Wenn man weiß, erst in zwei oder drei Jahren eine erstinstanzliche Entscheidung zu bekommen, wird man sich um außerjuristische Lösungen bemühen. Identische Rechtsnormen bewirken so Unterschiedliches. Im Westen mögen sie weithin durchgesetzt werden, im Osten sind sie nur eine mehr oder weniger wichtige Rahmenbedingung bei der Aushandlung von Kompromis$\operatorname{sen} .^{9^{8}}$

\section{Arbeitsbeziebungen unter Krisenbedingungen}

\section{Die Wirtschaftskrise}

Im Vergleich zum Jahr 1989 ist das Bruttosozialprodukt auf dem Gebiet der ehemaligen DDR bis Ende März 199 I auf etwa die Hälfte gesunken. ${ }^{99}$ Besonders betroffen ist die Industrie, in der 58 Prozent aller Erwerbstätigen beschäftigt sind. ${ }^{100}$ Über die Ursachen besteht wenig Streit. Durch die Herstellung der Währungs-, Wirtschaftsund Sozialunion am r.7. 1990 wurde die DDR-Wirtschaft von einem Tag auf den anderen der bundesrepublikanischen wie der Weltmarktkonkurrenz ausgesetzt. Ir-

93 Statistisches Jahrbuch für die Bundesrepublik Deutschland 1990, 340.

94 Statistisches Jahrhuch der DDR 1990, 447.

95 Berg NZA x990, 20

96 Zur Ersetzung der Konfliktkommission durch Schiedsstellen s. oben II I.

97 Dem Verfasser wurden Fälle bekannt, in denen über völlig eindeutige Klagen bewußt nicht entschieden wurde, weil der Richter befürchtete, als «SED-Freund " abgestempelt zu werden und so jede Chance auf Übernahme zu verlieren. Zur Situation der Richter s. weiter Behlert KJ 199t, 18sff. Inzwischen steht fest, daß weniger als die Hälfte aller DDR-Richter übernommen wurden - s. Südwestpresse vom 24. 9. 1991 S. 2.

98 S. weiter E. Peter KJ 1990, $481 \mathrm{f}$.

99 Vgl. Altvater Blatter für deutsche und internationale Polittk 1991, 668; Biedenkopf GMH 1991, 275; Thierse GMH 1991, 263. Weitere Angaben bei Kromphardt/Bruno-Latocha GMH 1991,734 100 Biedenkopf GMH 1991, 275. 
gendwelche Übergangsfristen, wie sie etwa den schwächeren EG-Partnern bei deren Beitritt eingeräumt wurden, kamen nicht in Betracht. Zusätzlich verschärft wurde die Situation durch den Zusammenbruch des RGW-Handels. Zahllose Vertragsbeziehungen wurden innerhalb weniger Monate beendet, weil die östlichen Auftraggeber nicht mehr leistungsfähig waren oder es vorzogen, ihre West-Devisen für Waren aus anderen Ländern auszugeben. Auch die Binnennachfrage brach zunächst in vielen Bereichen bis hin zu landwirtschaftlichen Produkten zusammen, da die DDR-Bevölkerung Westwaren selbst dann bevorzugte, wenn diese qualitativ keineswegs besser waren. Für die alte Bundesrepublik bewirkte dieser "Nachfragestoß" verstärktes Wachstum. ${ }^{101}$

Andere Formen der wirtschaftlichen Vereinigung wären denkbar gewesen, doch wurden sie von keinen politisch relevanten Kräften ins Auge gefaßt. In der DDR existierte keine nationale Bourgeoisie, die sich gegen einen Ausverkauf hätte wehren können. ${ }^{102}$ Für die große Mehrzahl der Bevölkerung war die sofortige Einheit mit der Erwartung verbunden, in recht kurzer Zeit den Lebensstandard der alten Bundesrepublik zu erreichen. Auch wenn sich dies zunächst als illusorisch erwies, hatte der Beitritt im Gegensatz zu einem langsamen Anpassungsprozeß einer selbständig bleibenden DDR den Vorzug, unmittelbar am Reichtum der Bundesrepublik partizipieren zu können: Die Verfassung verlangt die Herstellung von (wie auch immer zu definierenden) »einheitlichen Lebensverhältnissen «. Man war nicht mehr auf das Entgegenkommen ciner befreundeten Regierung angewiesen, die Jahr für Jahr hohe Zahlungen hätte leisten müssen. Aus Sicht der alten Bundesrepublik wäre der wirtschaftliche Gesamtaufwand für einen langsamen Anpassungsprozeß wohl vergleichbar hoch gewesen. I 20-1 50 Mrd DM pro Jahr zu bezahlen ${ }^{103}$, sind Regierung und Wirtschaft nur bereit, wenn sie selbst über den Gang der Dinge entscheiden können und nicht etwa damit potentielle Konkurrenten finanzieren. Die Zerschlagung der DDR-Industrie erschien unter diesen Umständen als das kleinere Übcl.

Die sich schnell vollziehende Deindustrialisierung sowie die Entlassungen im öffentlichen Dienst ${ }^{104}$ kommen in der Arbcitslosenstatistik nur unvollkommen zum Ausdruck. Nach eincm Bericht der Bundesanstalt für Arbeit gab es Ende August 1991 I,063 Mio Arbeitslose und $x, 45$ I Mio Kurzarbeiter. ${ }^{109}$ Bei einer Gesamtzahl von 9,2 Mio Erwerbstätigen zum Zeitpunkt der Maueröffnung ${ }^{106}$ entspricht dies einem Anteil von nur 27,3 Prozent. Die Proportionen ändern sich, wenn man weitere Gruppen berücksichtigt. Ende August 1991 waren 26r 000 Personen in befristeten ABMMaßnahmen beschäftigt ${ }^{107}$, und 280000 befanden sich in ebenfalls befristeten, vom Arbeitsamt geförderten Weiterbildungsmaßnahmen. ${ }^{108} 372000$ Beschäftigte haben vom sog. Vorruhestand Gebrauch gemacht ${ }^{\mathrm{s} 09}$. Die Zahl der Pendler, die in der alten Bundcsrepublik und Westberlin arbeiten, jedoch weiter im Osten wohnen, wird auf 350000 geschätzt ${ }^{10}$, etwa 400000 Arbeitskräfte sollen allein im Jahre 1990 in den Westen abgewandert sein. "' Nimmt man alle diese Gruppen hinzu, ergibt sich eine

I01 Das Bruttosozialprodukt wuchs in der alten Bundesrepublik im Jihre 1990 um 4,s Prozent, im Jahre I991 um 3,2\% (Bispinck/WSI-Tarifarchiv WSI-Mitc. 1992, I35).

102 Insoweit besteht eine - wenig beachtete - Parallele zu anderen ehemals sozialistischen Landern.

103 Vgl. die für 1991 errechneten Transfericistungen - s. Michael Schneider GMH 1991, $44^{8}$.

${ }_{104}$ Zu den Möglichkciten des Personalabbaus im offendichen Dienst s. oben III.

105 Handelsblatı vom 5.9. 1991 , S. 1.

106 Bispinck/WSI-Tarifarchiv WSI-Mitt. 1991,467

107 Am 31. 12. 1991 waren rund 390000 Personen über ABM beschäftigt: Muiller-Roden NZA 1992, 399.

108 Bispinck/WSI-Tarifarchiv $199 \mathrm{I}, 467$.

109 Bispinck/WSI-Tarifarchiv $1991,467$.

t10 Bundesanstalt fur Arbeit, Handelsblatt vom 5.9.1991, S. t.

11r Sund WSI-Mitt. 1991, 260. Nach Kromphardv/Bruno-Lacocha GMH 1991, 742 sicdelten zwischen 
Gesamtzahl von 4,297 Mio. Da es Ende 1989 in der DDR noch 8,547 Mio Erwerbstätige gab ${ }^{12}$, bedeutet dies, daß um die so Prozent der ursprünglichen Erwerbstätigen aus dem normalen Arbeitsprozeß in der früheren DDR ausgegliedert wurden.

In der alten Bundesrepublik verlief die Entwicklung genau gegenläufig. Ende August r99r war die Arbeitslosigkeit von 6,9 Prozent im entsprechenden Vorjahresmonat auf 6,2 Prozent gesunken"'3. Gleichzeitig wurden von Mitte 1990 bis Mitte 199I 800000 Arbeitsplätze neu geschaffen ${ }^{14}$, was nicht zuletzt auch Pendlern und Übersiedlern zugute kam. Entscheidender Anlaß war die aus der früheren DDR kommende Nachfrage.

Von der (registrierten) Arbeitslosigkeit sind Frauen überproportional stark betroffen. Ihr Anteil an den Erwerbstätigen belief sich in der DDR 1989 auf 48,88 Pro-

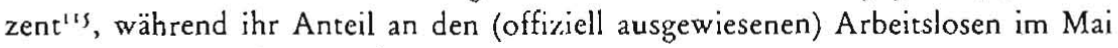
1991 s6,6 Prozent betrug. ${ }^{116}$

Arbeitslosigkeit ist nach 40 Jahren Beschäftigungssicherheit für die Betroffenen cinc neuartige und schwer zu verarbeitende Erscheinung. Dazu komme, daß der Arbeitsplatz im Leben des DDR-Bürgers eine noch erheblich wichtigere Stellung als in der (alten) Bundesrepublik hatte; schon wegen der Wochenarbeitszeit von in der Regel $43 \%$ Stunden und der oft langen Anfahrtswege spielte sich das Leben mehr am Arbeitsplatz als in der Freizeit ab. "7 Es überrascht daher nicht, daß eine vom Bundesministerium für Frauen und Jugend in Auftrag gegebene Studie zum Ergebnis kam, eine Alternativrolle als Nur-Hausfrau werde von der übergroßen Mehrheit der Frauen in der früheren DDR nicht akzeptiert; das Ministerium sieht hier ein erhebliches Konfliktpotential. ${ }^{18}$

\section{Tarifpraxis}

Die unterschiedlichen wirtschaftlichen Bedingungen schließen es aus, einheitliche Tarifverträge auf nationaler Ebene zu schließen. In der Praxis gibt es eine scharfe Trennung zwischen den Tarifgebieten in der alten Bundesrepublik und denen in der chemaligen DDR.

Die in praktisch allen Branchen neu abgeschlossenen Lohntarife' '9 haben zunächst den sog. Grundlohn auf einen bestimmten Prozentsatz des Westniveaus angehoben. Während die Nominalbeträge bei Beginn der Währungs-, Wirtschafts- und Sozialunion bei etwa einem Drittel lagen, belaufen sie sich nunmehr auf so bis 70 Prozent des in West-Tarifen Vorgesehenen ${ }^{120}$. In einzelnen Branchen wie der Metall- und der Druckindustrie ist ein Stufenplan vereinbart worden, wonach das Westniveau bis

Maueröffnung und Wahrungsunion 540000 Personen in den Westen über, von denen 300000 einen Arbeitsplatz fanden.

112 Statistisches Jahrbuch für die Bundesrepublık Deutschland 1990, 642

113 Bundesanstalt fur Arbeit, Handelsblat vom 5. 9.1991, S. 1.

114 Kromphard//Bruno-Latocha GMH 1991, 742.

II Statistisches Jahrbuch fur die Bundesrepublik Deutschland 1990, 642.

116 ANBA 1991, 959. Die ubrigen Gruppen einschließlieh der Kurzarbeiter sind nicht nach Geschlechtern aufgeschlussclt.

117 Bunke GMH 1991, 440; Biedenkopf GMH 1991, 276. Weidenfeld-Lorte (Aus Politik und Zeitgeschichte, $\mathrm{Nr}$. B 32/91, S. 7 ) berichten von einer Umfrage, wonach erheblich mehr Ost- als Westdeutsche die Arbeit "zu den liebsten Stunden des Tagess xahlten.

118 Bundesministerium für Frauen und Jugend ( $\mathrm{H} r \mathrm{rg}$.), Materialien zur Frauenpolitik 11/1991: Frauen in den ncuen Bundeslindern im Prozeß der deutschen Einigung. Frgebnisse einer repräsentativen Umfrage des Instituts fur angewandte Sozialwissenschaft Bad Godesberg, I:chruar 1991, S. 27.

119 Überblick einschließlich Ost-West-Verglcich bei Bispinck/WSI-laniarchiv WSI-Mitt. 1991, 472f. und WSI-Mitt. 1992, 121 .

120 Bispinck/WSI.Tarifarchiv WSI-Mitt. 1992, 126 
1994 oder 1995 erreicht werden soll. ${ }^{121}$ Sehr viel weniger erfolgreich war die Tarifpolitik bei den im Westen weit verbreiteten Zuschlägen und sonstigen Nebenleistungen: Werden auch sie berücksichtigt und beachtet man weiter, daß in der (alten) Bundesrepublik häufig übertarifliche Löhne bezahlt werden, so liegt das Lohnniveau in der ehemaligen DDR nur bei etwa 40 Prozent $^{122}$. Im öffentlicheen Dienst (einschließlich Bahn und Post) lagen die Verhältnisse zunächst ähnlich: Zwar wurden 60 Prozent der West-Gehälter tariflich festgeschrieben, doch wurden die in DDR-Zeiten erbrachten Arbeitsleistungen nicht angerechnet, so daß sich auf der Basis niedrigerer "Lebensaltersstufen « nur ein Niveau von etwa so Prozent ergab. Ende 1991 wurde auf entschiedenen Druck der Mitgliedschaft die volle Anrechnung der Vordienstzeiten erreicht, ${ }^{123}$ doch blieben bestimmte als ssystemnah « qualifizierte Personengruppen ausgeklammert. ${ }^{24}$

Aus der Bundesrepublik weithin übernommen wurden die sog. Rahmen- und Manteltarifverträge, in denen u. a. Fragen der Eingruppierung geregelt sind. Sie führten zu einer erheblich stärkeren Differenzierung zwischen den Beschäftigten ${ }^{125}$. Die Wochenarbeitszeit ist in der Regel auf 4o Stunden herabgesetzt worden, eine Annäherung an den West-Durchschnitt von 38 Stunden ist geplant. Eine entsprechende Entwicklung vollzieht sich im Bereich des Erholungsurlaubs. ${ }^{126}$

Die gewerkschaftlichen Forderungen lassen sich derzeit noch nicht mit steigender Produktivität rechtfertigen. Hauptargument ist der Gedanke einheitlicher Lebensbedingungen sowie die Verhinderung massenhafter Abwanderung in den besser verdienenden Westen. Wie lange diese Politik fortgesetzt werden kann, ist derzeit nicht absehbar.

\section{Verstärkte Staatsintervention}

Die Krise wird von staatlicher Seite auf zwei Ebenen bekämpft.

Zum einen werden Investitionen in den neuen Bundesländern erleichtert. Durch das Gesetz über die Spaltung der von der Treuhandanstalt verwalteten Unternehmen vom s. April $199^{127}$ wird erstmals im deutschen Gesellschaftsrecht die Möglichkeit geschaffen, große Unternehmenseinheiten in einzelne rechtlich selbständige Gesellschaften aufzuspalten. Dadurch erhöht sich die Chance, daß überlebensfähige Teile einzelner Kombinate und Betriebe von Investoren erworben und weitergeführt werden - freilich um den Preis, daß sich ein Interessent auch dann auf das "Filetstück" beschränken wird, wenn er an sich auch zum Erwerb der gesamten Einheit bereit gewesen wäre. Die in anderen westlichen Ländern recht eingehend geführte Diskussion um die Zerschlagung von Unternehmen durch Aufkäufer ("asset stripping «, "hostile take-over «) hat in der Bundesrepublik bisher so gut wie keine Resonanz. ${ }^{128}$ Weiter werden steuerliche Anreize geschaffen. Nach dem Steueränderungsgesetz vom 24. Juni $1991^{129}$ können Investitionen über die allgemeinen Regeln hinaus in den

121 Bispinck/WSI-Tarifarchiv WSI-Mitr. 1992, 127

$122 \mathrm{~S}$. die Gegenüberstellung der Effektivverdienste in der sachsischen und der bayerischen Metallindustrie bei Bispinck/WSI-Tarifarchiv WSI-Mitt. 1991, 474.

123 Dazu Jesse ZTR 1992, 91, der auch den Wortaut der geänderten Bescinmmungen wiedergibt.

124 Rechtliche Bedenken bei Jesse ZTR 1992, 97 und Däubler, Seniorität als Rechtsprinzip, (demnächst) in: FS Gnade, Köln 1992.

125 Backer, in: Kittner (Fn. 82), S. 338; Bispinck/WSI-Tarifarchiv WSI-Mitt. 1992, 125.

126 S. Fn. 119

$127 \mathrm{BGBl} .1,854$.

$128 \mathrm{~S}$. jedoch Hauschka-Roth $A G$ 1988, 18 I ff. und nunmehr Knoll, Die Ubernahme von Kapiralgesellschaften, Baden-Baden 1992. Zur *Abschreckungsfunktion* der Sozialplanpflicht s. Däubler, in: Daubler-Kittner-Klebe-Schneider (Hrsg.), BetrVG, 3. Aufl., Köln 1992, §11, Rn. 21. 129 BGBI. I, 1322. 
ersten s Jahren mit bis zu jo Prozent abgeschrieben werden. Außerdem bezahlte die

öffentliche Hand bis 30.6.1992 eine Investitionszulage von 12 Prozent (anschließend von 8 Prozent). Dazu kommen Förderprogramme des "Gemeinschaftswerks Aufbau Ost«. Weiter wurden durch das sog. Enthemmungsgesetz ${ }^{130}$ die Möglichkeiten verbessert, wie sich ein Investor gegen Rückerstattungsansprüche schützen kann. Das gesetzliche Instrumentarium dient insbesondere den Interessen neuer Unternehmen. Die bisher bestehenden können sich nur ausnahmsweise von der Bürde der Altschulden befreien und so kredit- und förderungswürdig werden: Nach den Gebräuchen der sozialistischen Planwirtschaft wurden Investitionen auch dann mit (billigen) Krediten finanziert, wenn das Unternehmen hohe Gewinne machte, die es an den Staatshaushalt abführte.' ${ }^{\prime \prime}$ Der Versuch, den vorprogrammierten Zusammenbruch vieler Altfirmen vor dem BVerfG unter Berufung auf Art. 3 Abs. I GG anzufechten, blieb erfolglos. ${ }^{132}$

Die zweite Ebene setzt am Arbeitsmarkt an. Schon vor der Herstellung der Einheit hatte die DDR ein eigenes Arbeitsförderungsgesetz erlassen ${ }^{133}$, das in $\$ 63$ Abs. 5 eine neue Art von Kurzarbeit schuf. Die Vorschrift blieb nach dem Einigungsvertrag erhalten; sie sollte ursprünglich am 30.6.199 I auslaufen, wurde dann aber bis 3r. 12.1991 verlängert. ${ }^{134}$

Nach dem in der (alten) Bundesrepublik geltenden $₫ 6_{3}$ Abs. 1 AFG gewährt das Arbeitsamt nur dann Kurzarbeitergeld, "wenn zu erwarten ist, daß durch die Gewährung ... den Arbeitnehmern die Arbeitsplätze und dem Betrieb die eingearbeiteten Arbeitnehmer erhalten verden. "Es geht daher anders als etwa in Italien'ss um eine typische Überbrückungsmaßnahme, die deshalb nach $\$ 67$ Abs. 1 AFG grundsätzlich auf 6 Monate befristet ist. Davon wich $\$ 63$ Abs. s AFG-DDR entscheidend ab: Das Kurzarbeitergeld wurde auch dann gewährt, wenn der Arbeitsplatz mit Sicherheit wegfallen würde. Gleichzeitig ist dic Stellung des Kurzarbeiters der des Arbeitslosen angenähert: Er muß grundsätzlich bereit sein, sich in jede andere zumutbare Arbeitsstelle vermitteln zu lassen, es sei denn, der Fortbestand seines Arbeitsplatzes sei zweifelsfrei gesichert. Weiter ist er auch verpflichter, an Weiterbildungsmaßnahmen teilzunehmen; andernfalls kann ihm für 8 Wochen das Kurzarbeitergeld gestrichen werden.

Eine weiterc arbeitsmarktpolitische Maßnahme liegt in der verstärkten Förderung von ABM-Maßnahmen. ${ }^{i 36}$ Arbeitslosen können danach im öffentlichen Interesse liegende Tätigkeiten zugewiesen werden, die andernfalls nicht oder nicht zu diesem Zeitpunkt erledigt würden ${ }^{137}$. Von Bedeutung ist dies insbes. im kommunalen Bereich und beim Ausbau der Infrastruktur. Die Betroffenen stehen in einem normalen Arbeitsverhältnis, das in der Regel auf ein bis zwei Jahre befristet ist. Nach den in der (alten) Bundesrepublik gemachten Erfahrungen ist die Chance für einen Übergang in ein Dauerarbeitsverhältnis allerdings außerordentlich gering. ${ }^{1{ }^{*}}$

I30 Vom 22. 3.1991, BGB1. I, 774 .

131 Kromphardr/Bruno-Latocha GMH i991, 738. Dem Verf. wurde von der Betriebsleıtung der Robotron $\mathrm{GmbH}$ Meiningen micgeteilt, das Kombinat Robotron habe von 1986 bis 1989 era/ 4 Mrd Mark Geswinn an den Staat abgefuhrt und erwa i Mrd Mark Investitionskreditc aufnehmen mussen. Nach der Wahrungsunion beliefen sich diesc aul soo Mio DM - Welcbes Unternehmen konnte solche Schulder zuruckhizahlen?

132 BVerfG DtZ 1991, 376.

133 AFG vom 22. 6. $1990, \mathrm{~GB}$. I, 403.

134 Geset\% zur Ánderung arbeusförderungsrechthicher und anderer sozialrechtlicher Vorschriften vom 21. 6, 1991, BGBI. 1, 1306, Arr. 2.

135 Dazu Ghezzi-Romagnoli, Il rapporto di lavoro, seconda edizione, Bologna 1987, \$\$339ff.

136 Zahlenangaben bei Múller-Roden NZA 1992, 399: Am 31. 12.1991 waren rund 390000 Personen er$\mathrm{faBt}$.

13) Einzelheiten in $\$ 9$ if. AFG und 9I If. AFG-DDR

138 Laatz-Jauss WSI-Mitt. 1989, 40: is Prozent wurden übernommen. 
Dritter Schwerpunkt der Arbeitsförderung sind Weiterbildungsmaßnahmen ${ }^{139}$; die Umstellung auf neue Arbeitsformen und Techniken ist in vielen Fällen nur aufgrund sachkundiger Anleitung möglich.

Schließlich dient das Altersübergangsgeld der Entlastung des Arbeitsmarkts. $₫ 249 \mathrm{e}$ AFG gewährt es allen arbeitslosen Erwerbspersonen, sofern sie das 55 . Lebensjahr erreicht haben. Die Regelung ist auf das sog. Beitrittsgebiet beschränkt und gilt zunächst bis 31. 12. 1992. Die Zahl der "Vorruheständler" belief sich Ende 1991 auf $705000 .^{14^{\circ}} \mathrm{Für}$ die Tarifpolitik oder - allgemeiner - für das System der industriellen Beziehungen sind beide Maßnahmekomplexe eher förderlich. Bei der Unterstützung von Investitionen ist dies nur beim Spaltungsgesetz anders; es kann den Eintritt das Arbeitsloswerden beschleunigen. Die Erweiterung und Umgestaltung der Kurzarbeit verschiebt den Eintritt der Arbeitslosigkeit und läßt in gewissem Umfang betriebliche Solidarzusammenhänge bestchen. Arbcitnehmer in ABM-Stellen und Weiterbildungsmaßnahmen können Mitglied der Gewerkschaft bleiben oder werden, doch laufen Tarifpolitik und betriebliche Mitbestimmung leer: Der Umfang der Arbeitgeberleistung bestimmt sich ausschließlich nach der staatlichen Förderung. Der formale Arbeitgeber ist atypisch insofern als er keinerlei finanziellen Spielraum besitzt.

\section{Zentralisierung von Verhandlungen auf Kosten der betrieblichen Ebene}

Das praktische Funktionieren der betrieblichen Mitbestimmung ist dadurch entscheidend beeinträchtigt, daß das einzelne Arbeitgeberunternehmen auch außerhalb der beschriebenen Maßnahmen einen sehr geringen Spielraum besitzt - sei es, daß die Treuhandanstalt als Alleineigentümerin detaillierte Vorgaben macht, sei es, daß die wirtschaftliche Situation keinerlei Zugeständnisse erlaubt. Besonders fühlbar wird dies bei Sozialplanverhandlungen, die angesichts des Personalabbaus in fast allen Betrieben geführt werden.

Die rechtliche Ausgangsposition des Betriebsrats ist nicht schlecht: Erweist sich der Arbeitgcber als nicht verhandlungs- und kompromißbereit, kann die Einigungsstelle angerufen werden, die unter einem neutralen Vorsitzenden entscheidet. ${ }^{141}$ Nach verbreiteter Auffassung hat sie dabei nicht nur die wirtschaftlichen Verhältnisse des Einzelunternehmens, sondern die des gesamten Konzerns zugrunde zu legen ${ }^{142}$; entscheidend käme es daher auf die wirtschaftliche Situation der Treuhandanstalt an.

Auf der anderen Seite kann die Arbeitgeberseite die Bestellung des Einigungsstellenvorsitzenden eine Reihe von Monaten hinauszögern. Außerdem hat sie die Möglichkeit, einen gegen ihren Willen zustande gekommenen Sozialplan vor dem Arbeitsgericht anzufechten, ein Verfahren, das sich über viele Jahre erstrecken würde. Erst nach seinem Abschluß könnten die Beschäftigten damit rechnen, tatsächlich Abfindungen oder andere Sozialplanleistungen zu crhalten.

Angesichts dieser wenig günstigen Perspektive kamen viele Verhandlungen nicht voran oder führten zu einer für die Arbeitnehmer außerordentlich unbefriedigenden

139 Zahlenangaben oben Fn. 108.

140 Bispinck/WSI-Tarifarchis WSI-Mitt. 1992, 121.

$141 \mathrm{Zu}$ Errichtung und I'unkcionsweise der Finigungsstelle s. Fitting-Auffarth-Kaiser-Heither, BetrVG, Handkommentar, 16. Aufl. $1990 \$ 76$ Rn. sff.

142 Hanau ZfA 1974, ros; Henssler, Der Arbeitsvertrag im Konzern, Berlin 1983, S. 146; Ohl, Der Sozialplan. Recht und Praxis kompensatorischer Leistungen für den Verlust des Arbeitsplaczes, Karlsruhe 1977, S. 6z; Richardi, Sozialplan und Konkurs, Düsseldorf r975, S. 84; Zollncr, Schweizerische Beitrage 7.um E.uroparecht, Bd. 14 , Genive 1974, S. 216 ; ähnlich im Bereich der betrieblichen Altersversorgung BAG DB 1989,1876 . 
Minimalabfindung. Gleichzeitig begann sich Anfang r991 öffentlich Unmut zu regen; die Montagsdemonstrationen in Leipzig wurden wieder aufgenommen. Angesichts dieser Rahmenbedingungen wurde eine politische Lösung auf höchster Ebene vereinbart. In einer "Gemeinsamen Erklärung " von DGB, DAG und Treuhandanstalt wurden Grundsätze für die Bemessung von Sozialplanleistungen und ihre Finanzierung durch die Treuhandanstalt festgelegt. ${ }^{143}$ Angesichts der bisherigen Praxis in der (alten) Bundesrepublik ist dies ein geradezu unerhörter Vorgang. Zum einen gehört die Verhandlung über einen Sozialplan zu den zentralen Kompetenzen des Betriebsrats; sie in der entscheidenden Frage des Finanzvolumens auf die Gewerkschaft zu übertragen, käme niemandem in den Sinn. Zum zweiten verhandelt für die Arbeitnehmerseite in allen Fällen die Branchengewerkschaft; dem DGB kommt nach sciner Satzung keine Tariffähigkeit zu. Dennoch hat er im vorliegenden Fall die entscheidenden Weichen gestellt und eine Art »interprofessionelles Spitzenabkommen « abgeschlossen. Zwar handelt es sich nicht um einen Tarifvertrag oder eine sonstige rechtlich verbindliche Abmachung (auch dies eher eine Seltenheit), doch gingen beide Seiten davon aus, daß die Erklärung effektiv beachtet wird. ${ }^{144}$ Soweit ersichtlich, ist dies auch geschehen. Im Augenblick (Juni 1992) erfolgt eine Auflokkerung in der Richtung, daß abweichende "Regelungen « mit Einzelgewerkschaften getroffen werden, die in der Regel bessere Leistungen vorsehen. Eine gewisse Rückkehr zur Normalität zeichnet sich ab.

\section{Die Vereinbarung über die sog. ABS-Gesellschaften}

Die traditionellen Formen der Interessenvertretung wurden erst recht verlassen, als am I7. Juli I991 die "Rahmenvereinbarung zur Bildung von Gesellschaften zur Arbeitsförderung, Beschäftigung und Strukturentwicklung (ABS) « geschlossen wurde. An ihr waren DGB und Einzelgewerkschaften, Arbeitgeber, Treuhandanstalt und die Regierungen der neuen Bundesländer sowie des Landes Berlin beteiligt. Auch inhaltlich wird Neuland betreten. Die ABS-Gesellschafren, die es in einer Reihe von Städten schon vorher gab ${ }^{245}$, sollen durch aktiven Einsatz von Arbeitslosen oder durch Arbeitslosigkeit bedrohten Beschäftigten den notwendigen Strukturwandel erleichtern. Gedacht ist insbes. an die systematische Durchführung von Qualifizierungsmaßnahmen, an Arbeiten zur Verbesserung der Infrastruktur und zur Sanierung von Unternehmen sowie an Planungstärigkeiten, die sich auf den Abbau ökologischer Belastungen wie auch auf die Ansiedlung neuer Industrien oder die Förderung von Handwerksbetrieben beziehen können. Träger soll eine als gemeinnützig anerkannte $\mathrm{GmbH}$ oder ein gleichfalls gemeinnütziger eingetragener Verein sein. ${ }^{146}$ ABS-Gesellschaften sollen der Rahmenvereinbarung entsprechend sowohl auf lokaler wie auch auf regionaler und auf Landesebene errichtet werden. Die Finanzierung geschieht im wesentlichen aus öffentlichen Mitteln. Weiterbildung und im Allgemeininteresse liegende Arbeiten werden von der Arbeitsverwaltung gefördert; dazu kommen Programme der Landesregierung. Mit weiterexistierenden Betrieben sind Kooperationsabkommen vorgesehen, wonach beispielsweise Räume und Arbeitsgeräte zur Verfügung gestellt werden. Auch soll von dort oder aus dem Westen Manage-

143 Einzelheiten bei Däubler AiB 1991, 179 ff. und Hanau, in: Hommelhoff(Hrsg.), Treuhandunternehmen im Umbruch, Köln 1991, S. 103 ff.

144 Insoweir besteht durchaus eine Parallele zum icalienischen Accordo interconfederale, zum franzósischen accord interprofessionnel und zum spanischen acuerdo interconfederal.

I4s S. die Befragung von Stöber in: Hans-Böckler-Stiftung (Hrsg.), Qualifizierungs- und Beschäftigungsinitiativen in Ostdeutschland. Ein Leitfaden für die Praxis, Düsseldorf 1991, S. $30 \mathrm{ff}$.

146 Eine Mustersatzung ist abgedruckt in WSI-Mitt. 1991, 681. 
mentwissen übernommen werden. An den regionalen und auf Landesebene bestehenden Trägergesellschaften will sich auch die Treuhandanstalt kapitalmäßig beteiligen. Selbst die Gewerkschaften wollen einen Anteil übernehmen, der allerdings die symbolische Höhe von 5000 DM pro Gesellschaft nicht übersteigen darf.

Die Konzeption hat den Vorzug, die vorhandenen Kräfte zu bündeln und an den vor Ort entstehenden Problemen anzusetzen. Sie vermeidet teure Großprojekte ( $* \mathrm{Ka}$ thedralen in der Wüste $)$ und kann zur Entstehung von kleinen Dienstleistungs- und Handwerksbetrieben sowie der für Großbetriebe nötigen Infrastruktur beitragen. Für die Arbeitnehmerseite ergeben sich zumindest kurzfristig einige weitere Vorteile: Viele werden nicht in die Arbeitslosigkeit geschickt, sondern bleiben in betrieblichen Zusammenhängen. Nicht wenige behalten auch den Arbeitsvertrag zu ihrem bisherigen Arbeitgeberunternehmen, der solange suspendiert und durch ein Arbeitsverhältnis zur ABS-Gesellschaft ersetzt wird, bis die Umstrukturierung erfolgreich abgeschlossen ist. Auch bleiben die tariflichen Arbeitsbedingungen grundsätzlich erhalten. ${ }^{147}$ Auf der anderen Seite handelt es sich nur um eine - nicht eben billige - Übergangsmaßnahme; schon die geplante Reduzierung von $\mathrm{ABM}^{14^{8}} \mathrm{kann}$ vielen Beschäftigungsgesellschaften einen schweren Schlag versetzen.

\section{Ausblick}

Das gesetzliche Arbeitsrecht der Bundesrepublik hat die völlig neuartige Situation in der früheren DDR relativ unbeschadet überstanden. Von Defiziten beim gerichtlichen Rechtsschutz abgesehen, hat es sich in den neuen Bundesländern fest etabliert. Der Gesetzgeber hat kein "Notstandsarbeitsrecht" geschaffen - sieht man einmal von der Suspendierung des $\$ 6 r z a B G B$ im Insolvenzverfahren ab, die bis 31. I2. 1992 befristet ist ${ }^{149}$ und die wenig praktische Bedeutung besitzt, weil die Treuhandanstalt die "stille Liquidation " gegenüber dem Gesamtvollstreckungsverfahren (= Konkurs) vorzieht.

Das wirtschaftliche Gefälle schlägt sich in den Lohn- und Gehaltstarifen sowie in den relativ bescheidenen Sozialplänen nieder. Die Gemeinsame Erklärung zwischen den gewerkschaftlichen Spitzenverbänden und der Treuhand sowie die ABS-Vereinbarung stellen eine neue Form zentraler Arrangements dar, die vermutlich kaum Nachfolger finden werden. Die Verhandlungssysteme erwiesen sich als das flexible Element des Arbeitsrechts.

Die Rechtsprechung, die bislang vorwiegend in Form von Entscheidungen des ArbG Berlin und des LAG Berlin existiert ${ }^{150}$, hat die vorhandenen Unterschiede $z$ wischen West und Ost nicht gemildert, sondern eher akzentuiert. Wo noch keine festgefügten BAG-Aussagen vorlagen, hatte es - um es vorsichtig auszudrücken - die Arbeitnehmerseite schwer. Tarifverträge, die vor dem 1.7. 1990 geschlossen wurden, sollen nur dann wirksam sein, wenn sie gemäß dem damals noch geltenden $₫ 14$ AGB registriert wurden ${ }^{151}$ - ein wenig einleuchtendes Kleben an der formalen Rechtslage, obwohl die Behörden zum Teil nicht mehr funktionsfähig waren und alle Beteiligten mit einer unmittelbar bevorstehenden Einführung des TVG rechneten. Nicht besser soll es auch Sozialplänen aus der Zeit vor der Währungsunion ergehen; nach Auffassung

\footnotetext{
I47 Einzelfragen bei Kaiser NZA 1992, 193.

I 48 Müller-Roden NZA 1992, $399 \mathrm{ff}$

149 \16 Abs. 2 Spaltungsgesetz.

150 Dies gilt jedenfalls fur die veroffentlichten Entscheidungen. Die Ursache liegt vermutlich darin, daß die Westberliner Arbeitsgerichtsbarkeit lediglich ihre Zuständigkeit auf Ostberlin ausdehntc und so von Anfang an funktionsfähige Arbeitsgerichte vorbanden waren.

Is। BAG DB x992, 481; anders Berirksgericht Rostock LAGE AGB 1977 Nr. 14
} 
des LAG Berlin's2 sind sie unwirksam und auch nicht in eine abgestimmte Änderung der Arbeitsverträge umzudeuten. Wer in der Vergangenheit als Mitglied einer LPG Arbeit leistete, war trotz Weisungsgebundenheit kein Arbeitnehmer und unterlag deshalb nicht dem Arbeitsrecht. ${ }^{153}$ Arbeitsverhältnisse von Rentnern mit der öffentlichen Hand endeten automatisch mit dem 3. 10. 1992, obwohl dies nach dem Wortlaut des Einigungsvertrags alles andere als zwingend ist. ${ }^{154}$ Kollektivverträge, die nach DDR-Recht die Steuerfreiheit bestimmter Zusatzleistungen konstitutiv festlegen konnten, sind bei Weitergeltung nicht als "Nettolohnvereinbarungen « auszulegen, "ss obwohl dies den Absichten der Beteiligten am ehesten entsprechen würde. Wenig Sensibilität entwickelte das LAG Berlin für die Situation der Arbeitnehmer im Beitrittsgebiet: Wer sich auf die Rechtsauskunft seines Betriebsrats verlasse, handle schuldhaft und könne keine nachträgliche Zulassung seiner Kündigungsschutzklage nach $₫ s \mathrm{KSch} G$ verlangen; die betriebliche Interessenvertretung sei keine »zuverlässige und berufene Stelle ${ }^{156}$ Es paßt ins Bild, daß die Berliner Arbeitsgerichte Osttarife auch dann anwenden, wenn einzelne Arbeitnehmer auf Dauer in den Westteil der Stadt versetzt wurden. '"7 Das BAG wird darauf zu achten haben, daß bei "OstSachverhalten " keine anderen Maßstäbe als bei "West-Sachverhalten " angelegt werden. Zusammen mit den Sonderkündigungsgründen für den öffentlichen Dienst, die auf Initiative des Freistaats Sachsen bis zum 31. x2.1993 verlängert wurden ${ }^{158}$, entstünde sonst unter der Hand eine Art "Arme-Leute-Arbeitsrecht «, das uns noch weiter von der viel beschworenen Einheitlichkeit der Lebensverhältnisse wegführen würde.

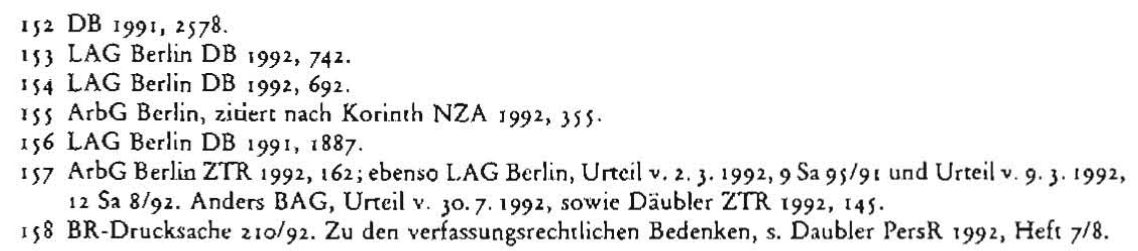

